\title{
Normen als Ordnungsrahmen der Friedensmediation
}

\author{
Anne Holper (D) - Lars Kirchhoff · Felix Würkert
}

Eingegangen: 28. Oktober 2019 / Überarbeitet: 26. März 2021 / Angenommen: 12. April 2021 / Online publiziert: 31. Mai 2021

(C) Der/die Autor(en) 2021

Zusammenfassung Die gestiegenen normativen Ansprüche an Friedensmediation werden von Praktiker*innen bisweilen als verwirrend und einengend wahrgenommen. Bei genauer Betrachtung der Normen und der darin formulierten Verhaltenserwartungen lassen sich diese jedoch präzise definieren und bieten weitaus mehr Spielraum als vermutet. Mehr noch, sie lassen sich in der Vermittlungspraxis als wertvolles Orientierungssystem nutzen, mit dem im Idealfall verhindert werden kann, dass Friedensprozesse und -vereinbarungen scheitern, schaden oder rechtlich nichtig sind. An einigen Stellen steht allerdings noch eine dezidierte Übersetzung dieses Wissens

In diesem Artikel bereiten die Autor*innen das von ihnen als Mitglieder der Initiative Mediation Support Deutschland entwickelte und vom Auswärtigen Amt herausgegebene Fact Sheet ,Normativer Bezugsrahmen und völkerrechtliche Grundlagen der Friedensmediation“ (AA und IMSD 2017) wissenschaftlich auf. Einzelne Abschnitte wurden mit Einverständnis des Auswärtigen Amtes im Wortlaut bzw. mit leichten Umformulierungen übernommen.

In this article the authors provide a scientific account of the content of "The normative framework and the international legal basis of peace mediation" (AA and IMSD 2017), a Fact Sheet which they developed as members of the Initiative Mediation Support Germany and which was published by the Federal Foreign Office in 2017. Certain sections have been reproduced with the permission of the Federal Foreign Office, either in full or with slight rewording.

\footnotetext{
A. Holper $(\triangle)$

Center for Peace Mediation, Europa-Universität Viadrina, Große Scharrnstraße 59, 15230 Frankfurt (Oder), Deutschland

E-Mail: holper@europa-uni.de

L. Kirchhoff

Institut für Konfliktmanagement, Center for Peace Mediation, Europa-Universität Viadrina, Große Scharrnstraße 59, 15230 Frankfurt (Oder), Deutschland

E-Mail: kirchhoff@europa-uni.de

F. Würkert

Lehrstuhl für Öffentliches Recht, insbesondere Völkerrecht und Europarecht,

Helmut-Schmidt-Universität der Bundeswehr in Hamburg, Holstenhofweg 85, 22043 Hamburg,

Deutschland

E-Mail: felix.wuerkert@hsu-hh.de
} 
in die praktische Handlungslogik und faktischen Abläufe von Friedensprozessen sowie eine strukturierte Vorgehensweise aus, mit der sich die zentralen normativen Dilemmata handhaben lassen, die der Friedensmediation inhärent sind.

Schlüsselwörter Ethik · Friedensmediation · Friedensprozesse $\cdot$ Normen · Methodik · Völkerrecht

\section{Norms as a regulatory framework for peace mediation}

Abstract Practitioners often perceive the increased normative demands on peace mediation as confusing and constricting. On closer examination of the norms and the behavioural expectations formulated therein, however, these expectations can be precisely defined and offer far more flexibility than assumed. Moreover, these norms can be used as a valuable orientation system for mediation practice that can ideally prevent peace processes and agreements from failing, causing harm or being legally invalid. At some points, however, there is still a need for a focused translation of this knowledge into the practical logic and operative procedures of peace processes as well as for a structured approach to deal with the central normative dilemmas inherent in peace mediation.

Keywords Ethics $\cdot$ Peace mediation $\cdot$ Peace processes $\cdot$ Norms $\cdot$ Methodology . International law

\section{Einleitung}

Der normative Rahmen der Friedensmediation ${ }^{1}$ hat in letzten Jahren sowohl mit Blick auf das Verfahren als auch die Verhandlungsgegenstände stark an Sichtbarkeit und Kontur gewonnen (Hellmüller et al. 2017, 2015; Kastner 2015; Wählisch 2016). Auch wenn es weiterhin keinen einheitlich konsolidierten normativen Rahmen für die Friedensmediation gibt, wurden zahlreiche methodische, ethische und völkerrechtliche Bausteine genauer definiert und ausdifferenziert (AA und IMSD 2017, S. 2). ${ }^{2}$

Durch die Rezeption dieser Normen sind die Erwartungen mit Blick auf übergreifende Imperative und konkrete Qualitätsansprüche, die Mediationsprozesse und

\footnotetext{
1 „Mediation is a process whereby a third party assists two or more parties, with their consent, to prevent, manage or resolve a conflict by helping them to develop mutually acceptable agreements" (Vereinte Nationen 2012).

${ }^{2}$ Die UN-Guidance on Effective Mediation, deren Rezeption und Inhalt in Abschnitt 3. und 4. vorgestellt werden, stellt aus Sicht der Autor*innen (noch) keinen einheitlich konsolidierten normativen Rahmen in dem Sinne dar, dass von diesem nicht abgewichen werden könnte, ohne dafür eine rechtfertigende Begründung abzugeben. Die Guidance wurde in Resolution GA/65/283 einstimmig beim UN Generalsekretär in Auftrag gegeben und als Annex zum Bericht des UN Generalsekretärs vom 25. Juni 2012 (A/66/811, Strengthening the role of mediation in the peaceful settlement of disputes, conflict prevention and resolution) veröffentlicht.
} 
-vereinbarungen in (potenziell) gewaltsamen politischen Konflikten erfüllen sollen, erheblich gestiegen:

As mediation lies at the core of a wider peacebuilding process, an increasing number of actors in the peacebuilding field view mediation processes as an opportune time to promote international norms, such as inclusivity, gender equality, transitional justice and democracy. The UN Guidance for Effective Mediation (Vereinte Nationen 2012) defines certain principles as essential for more effective mediation, thereby confirming the growing imperative of normative frameworks in mediation. Consequently, mediators are often not only expected to facilitate processes aimed at ending hostilities between warring parties, but are asked to integrate a specific set of norms held by their mandate-givers into their mediation strategies (Hellmüller et al. 2017).

Da diese Entwicklungen noch jung sind, sind im Feld der Praktiker*innen allerdings oftmals noch kein ausreichendes normatives Wissen und auch keine etablierten Formate zur differenzierten Vermittlung dieses normativen Wissens vorhanden. Selbst eine umfassende schriftliche Darstellung des für Friedensmediation relevanten normativen Wissens fehlt.

Parallel dazu hat sich in Wissenschaft und Praxis die Grundsatzdebatte darüber intensiviert, welche Rolle Normen im Allgemeinen und völkerrechtliche Aspekte im Besonderen in der Friedensmediation einnehmen (sollten) - in Prozessen sowie in den in dort entstehenden Vereinbarungen (AA und IMSD 2017, S. 2): Dürfen Mediator*innen in dem rechtlich prekären, dafür umso mehr machtpolitisch-militärisch dominierten Raum internationaler Konflikte ausnahmsweise jenseits normativer Vorgaben agieren (Coleman 2015; Coady 2014; Walzer 1986) - oder müssen sie sich gerade in solchen Konstellationen an bestimmte Normen halten bzw. sogar aktiv für deren Einhaltung sorgen (Hellmüller et al. 2017; Kastner 2015)? Wie können Normen mit tendenziell universalistischem, die existierende kontextuelle bzw. kulturelle Diversität nicht abbildendem Geltungsanspruch kontext- bzw. kulturadäquat in Friedensprozesse eingebracht werden (Zeller und Schellekens 2017; Convergne 2016)? Wie sollte priorisiert werden, wenn Imperative aus verschiedenen Normensystemen kollidieren (z. B. international propagierte vs. lokal verankerte Normen; Beendigung der Gewalt vs. Wahrung von Menschenrechten (Palmiano Federer 2016))?

In der Debatte spiegeln sich zum einen Unklarheiten darüber, welche Normen an welchen Stellen überhaupt relevant werden und zum anderen unterschiedliche Ansichten darüber, welche Geltung diese Normen in der Praxis haben (sollten) und wie sie anzuwenden sind. Insgesamt besteht damit eine beträchtliche Diskrepanz zwischen den an Mediator*innen gerichteten normativen Erwartungen und ihrer praktischen Umsetzung bzw. Umsetzbarkeit. Berührungsängste vor einer schwer greifbaren Materie scheinen maßgeblich zu dieser Diskrepanz beizutragen, so der Eindruck der Autor*innen aus dem kontinuierlichen Austausch mit Friedensmediator*innen im Verlauf der letzten zehn Jahre, u. a. im Rahmen des Mediation Support 
Networks und der Initiative Mediation Support Deutschland ${ }^{3}$ sowie im internationalen diplomatischen Kontext.

Vor diesem Hintergrund haben die Autor*innen im Jahr 2017 das Fact Sheet „Normativer Bezugsrahmen und völkerrechtliche Grundlagen der Friedensmediation" zusammengestellt. Ziel des Fact Sheets war es, Praktiker*innen der Friedensmediation im deutschsprachigen Raum eine grundlegende Übersicht über die für das Feld relevantesten normativen Bausteine mit einem Fokus auf jus cogens Vorschriften ${ }^{4}$ sowie eine Abwägungshilfe für die in der Praxis oft auftretenden Normenkollisionen zur Verfügung zu stellen. Das für die Zwecke der Praxis im Fact Sheet stark komprimierte Wissen wird in dem vorliegenden Beitrag wissenschaftlich reflektiert und in aktuelle fachliche Diskussionsstränge eingebettet. Die Grundstruktur sowie einzelne Bestandteile des Fact Sheets sind dabei bewusst erhalten geblieben und letztere jeweils entsprechend kenntlich gemacht, um die sonst oft unverbundenen Diskurse von Praxis und Wissenschaft an dieser Stelle gezielt zusammenzuführen.

Wie das Fact Sheet will der Artikel zum einen Wissen darüber vermitteln, welche konkreten Erwartungen die schon lange bestehenden und die neu formulierten Normengefüge an die Praxis der Friedensmediation richten. Da die Gesamtschau völkerrechtlich relevanter materiell-rechtlicher Vorschriften im Fact Sheet bereits verfügbar ist, konzentriert sich dieser Artikel auf die wissenschaftliche Einbettung und Kontextualisierung der normativen Bezugsgrößen von Friedensmediation.

Zum anderen will der Artikel zur Klärung beitragen, welche Lösungsoptionen bei praktischen Anwendungsfragen bestehen. Die Autor*innen wollen damit zeigen, dass der normative Ordnungsrahmen für die zahlreichen komplexen, kontextgebundenen Entscheidungen, die zur Gestaltung von Mediationsverfahren und -vereinbarungen zu treffen sind, wertvolle Orientierung bieten kann - allerdings nur, wenn man diesen Ordnungsrahmen im Detail kennt und ihn mediations-methodisch zu nutzen weiß. Einfache Formeln für diese schwierigen Entscheidungen bieten die Normen nicht, aber ein Gerüst, Handlungsoptionen methodisch, ethisch und rechtlich abzuwägen und damit letztlich auch praktisch abzusichern (AA und IMSD 2017, S. 2).

Schließlich will der Artikel zu einer intensiveren wissenschaftlichen Auseinandersetzung der deutschsprachigen Friedens- und Konfliktforschung mit den Entwicklungen im Bereich der Friedensmediation im Allgemeinen und der Rolle von und dem Umgang mit Normen in diesem Feld im Besonderen anregen (Holper

\footnotetext{
${ }^{3}$ Das Mediation Support Network (MSN) ist ein internationales Netzwerk, in dem primär nichtstaatliche Organisationen aus dem Bereich Mediation und Mediationsunterstützung zusammenarbeiten, um den Wissensaustausch und die Abstimmung zu Entwicklungen und konkreten Prozessen der Mediation im Kontext bewaffneter Konflikte zu verbessern (https://mediationsupportnetwork.net). Die Initiative Mediation Support Deutschland (IMSD) ist ein Netzwerk deutscher Organisationen aus dem Bereich Mediation und Mediation Support, das darauf abzielt, die Instrumente Friedensmediation und Mediation Support als integralen Bestandteil der deutschen Außenpolitik zu stärken und weiterzuentwickeln (https://www. friedensmediation-deutschland.de).

${ }^{4}$ Zwingendes Völkerrecht (jus cogens) steht hierarchisch über anderen Normen des Völkerrechts und kann nur durch gleichrangiges späteres zwingendes Völkerrecht geändert werden. Es spiegelt die fundamentalen Werte der Internationalen Gemeinschaft wider und ist universal anwendbar. Wenn auch keine abschließende Liste besteht, fällt das u.a. das Recht auf Selbstbestimmung, das Verbot von Verbrechen gegen die Menschlichkeit und das Folterverbot darunter (International Law Commission 2019).
} 
und Kirchhoff 2020). Die enge Anlehnung an das vom Auswärtigen Amt herausgegebene Fact Sheet zielt dabei darauf ab, die Wissenschaft mit den normativen Herausforderungen des Feldes aus der Warte und in der Begrifflichkeit von Friedensmediationspraktiker*innen und politischen Entscheidungsträger*innen vertraut zu machen.

Der Artikel leistet damit eine Kategorisierung, Illustration, Kontextualisierung, Operationalisierung und Ausbalancierung von Normen als einem möglichen Ordnungsrahmen methodenbasierter Friedensmediation. Im ersten Schritt führt der Text auf Basis eines breiten Normbegriffs die unterschiedlichen in diesem Handlungskontext relevanten Normdimensionen zusammen (Kategorisierung). Im zweiten Schritt wird ein Blick auf die exemplarische Ausdifferenzierung dieses Ordnungsrahmens durch den ersten maßgeblichen Konsolidierungsversuch, der UN Guidance for Effective Mediation von 2012, und die im Zuge ihrer Etablierung kritisierten Aspekte geworfen (Illustration). Im dritten Schritt werden drei komplementäre rechtliche Referenzsysteme vorgestellt (akteursspezifische Normen, Transitional Justice, Rolle von Gerichten), die in unterschiedlichen Konfliktkonstellationen und -kontexten unterschiedliche Relevanz entfalten können (Kontextualisierung). Im vierten Schritt wird mittels Beispielen (Operationalisierung) aufgezeigt, wie eine methodisch fundierte Abwägung kollidierender Normen zu konkreten Ergebnissen führen kann (Ausbalancierung).

\section{Normbegriff und Normkategorien}

Normen sind naturgemäß Gegenstand beständiger wissenschaftlicher Aufmerksamkeit (Finnemore 2000). ${ }^{5}$ Die zentrale Eigenschaft von Normativität ist es, ein Sollen in Form einer ,,positiv markierte[n] Möglichkeit““ (Möllers 2015) zu formulieren. Nach einem konstruktivistischen Normenverständnis ist diese positiv markierte Möglichkeit nicht naturgegeben sondern sozial hergestellt und dadurch in vielfacher Weise kontext- und interaktionsabhängig (Björkdahl 2002). ${ }^{6}$ Aus Katzensteins Definition von Normen als ,collective expectations for the proper behavior of actors with a given identity“ (1996) erschließt sich zudem, a) dass es sich bei Normen im Allgemeinen immer erst einmal ,nur“ um Verhaltenserwartungen handelt (nicht um -vorschriften, die vielmehr nur einen Teil aller Normen ausmachen, siehe unten), b) dass diese Erwartungen kollektiv getragen sein müssen (um anders als individuelle Interessen soziale Geltung beanspruchen zu können) und sich c) auf identifizierbare Handelnde und Handlungen beziehen müssen (um anders als die auf allgemeine Wesens- und Haltungsmerkmale gerichteten Werte konkretes Verhalten anvisieren zu können). Ein kultursoziologischer Normenbegriff wie ihn Reckwitz

\footnotetext{
5 Die Definition des Normbegriffs bereitet erhebliche Probleme, insbesondere wenn diese eine Abgrenzung des Normativen vom Nicht-Normativen leisten soll (Möllers 2015). Eine abstufende Kategorisierung von Gewohnheit als Nicht-Norm, über Brauchtum, Sitte, moralische Regel bis hin zum Recht als Normen, findet sich gleichwohl bei Luhmann (1987). Auch Hart (1994) sieht Gewohnheiten als nicht normativ, diskutiert ansonsten jedoch „Regeln“.

${ }^{6}$ Eine kritische Differenzierung findet sich bei Wiener (2007).
} 
entwickelt, geht davon aus, dass Normen immer in kulturellen Sinnsystemen (im Sinne von kontextbezogenen Wissensordnungen) operieren, welche die Wirklichkeit der jeweiligen Akteure prägen, und daher stets kulturell bedingt sind (Reckwitz 2000, S. 84, 143 f.). Kultur als Oberbegriff für gruppenbezogene Bedeutungssysteme bezieht sich dabei etwa auf soziale, rechtliche, politische, institutionelle, religiöse oder professionelle Kulturen. Zusammengenommen versteht der Text Normen als kollektive Erwartungen in Bezug auf ein situativ und akteursabhängig „,angemessenes“ Verhalten, wobei das jeweils „Adäquate“ als ein sich stetig veränderndes und kulturbedingtes Konstrukt menschlicher Interaktion begriffen wird.

Im Rahmen von Friedensmediation gilt es, zahlreiche Verhaltensentscheidungen mit Blick auf das Verfahren und die Verhandlungsmaterie zu treffen, daher spielen Normen - ob explizit oder implizit - eine große Rolle in der praktischen Arbeit von Vermittler*innen. ${ }^{7}$ Da Mediationsprozesse im Einzugsgebiet unterschiedlicher normativer Bezugssysteme stehen, sind die Verhaltenserwartungen, die von Mandatgeber*innen, Vertreter*innen der internationalen Gemeinschaft und der betroffenen Bevölkerung an sie herangetragen werden, sehr unterschiedlicher Herkunft und Zielrichtung. Sie umfassen methodische Prinzipien (z. B. zum Design von Friedensprozessen und -vereinbarungen), ethische Grundsätze (z.B. zur Vermeidung von Schaden), politische Programmziele (z.B. Demokratisierung), institutionelle Regularien (z.B. interne Weisungen, Codes of Conduct), nationale und internationale rechtliche Rahmensetzungen (z.B. zum Schutz von Opfergruppen), und nicht zuletzt soziale und religiöse Normen (z.B. zu sozialen und Geschlechterhierarchien).

Wichtig ist daher die Unterscheidung zwischen unterschiedlichen Arten vermittlungsrelevanter Normen, etwa zwischen sozialen, politischen, religiösen, methodischen, ethischen oder rechtlichen Normen. Diese Normkategorien und ihre Bezugssysteme unterscheiden sich hinsichtlich ihres Bindungsanspruchs, ihrer Bindungswirkung und der Konsequenzen ihrer Nichtberücksichtigung: So beschreiben soziale, politische, kulturelle, religiöse, methodische und ethische Normen Verhaltenserwartungen, die nicht rechtlich einklagbar sind, deren Missachtung aber mit schwerwiegenden Sanktionen der jeweiligen Sphäre belegt sein kann. Rechtliche Normen stellen dagegen Verhaltensvorschriften oder institutionelle bzw. definitorische Regelungen dar, die häufig über einen rechtlichen Durchsetzungsmechanismus verfügen und beanspruchen, über allen anderen nichtrechtlichen Normen und Vereinbarungen zu stehen (AA und IMSD 2017, S. 2). Die Konsequenzen der Nichtberücksichtigung theoretisch aller genannten Normenarten sind beispielsweise Reputationsverlust oder ein Entzug der politischen oder gesellschaftlichen Unterstützung. Bei rechtlichen Normen treten bisweilen strafrechtliche Sanktionen, Erklärungen rechtlicher Unwirksamkeit und andere rechtliche Sanktionen hinzu (Finnemore 2000; McAdams 1997). Die Umsetzbarkeit und Nachhaltigkeit von Friedensprozessen und -vereinbarungen kann im Lichte dieser Konsequenzen folglich vielfach beeinträchtigt werden, wenn relevante Normen nicht beachtet werden.

\footnotetext{
7 Damit ist gemeint, dass eine Norm in einem Verfahren explizit adressiert werden kann, während sie in einem anderen unausgesprochen bleiben kann aber dennoch berücksichtigt wird, weil sie den Akteuren bekannt und bewusst ist.
} 


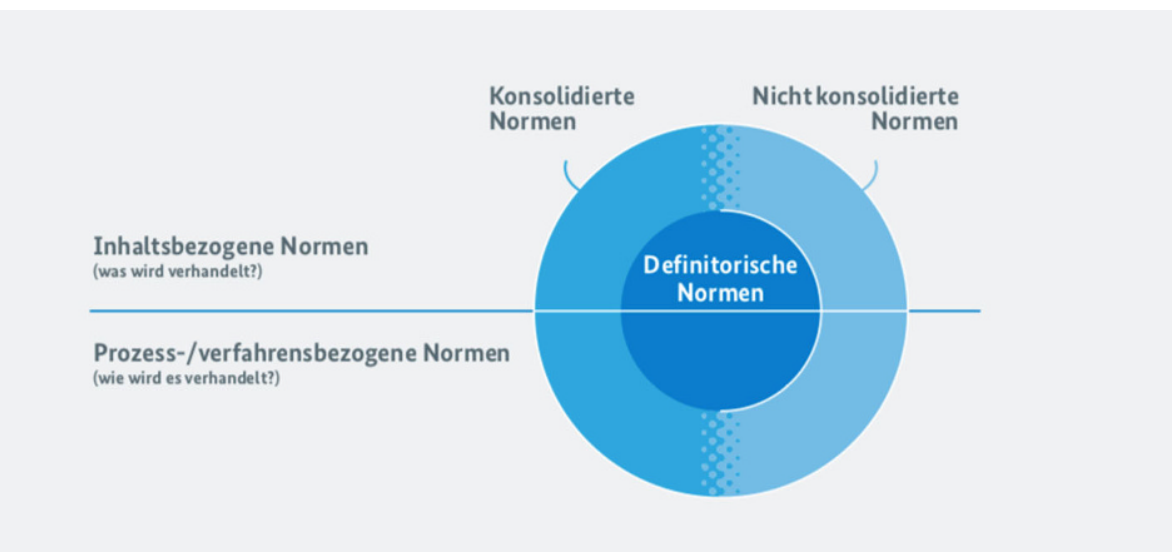

Abb. 1 Normen im Mediationskontext. (In Anlehnung an Grafik in AA und IMSD 2017, S. 3, nach Hellmüller et al. 2015)

Essentiell ist zudem eine Unterscheidung zwischen den Verfahrensnormen oder prozessbezogenen Normen der Friedensmediation (,Normen für die Friedensmediation") und den materiellen oder inhaltsbezogenen Normen, die den inhaltlichen Kern der Verhandlungen und Friedensvereinbarungen betreffen (,Normen in der Friedensmediation“) (AA und IMSD 2017, S. 2). Friedensvereinbarungen selbst wiederum können als normativ geprägte bzw. prägende Ergebnisse der Friedensmediation begriffen werden, die in letzterer Funktion den Konfliktkontext neugestalten (können). Bisweilen sind die Grenzen allerdings nicht trennscharf: während materiell-rechtliche Referenzrahmen oft ebenso den Prozess einer Vermittlung mitprägen, können Verfahrensnormen integraler Teil des Verhandlungsgegenstands sein (AA und IMSD 2017, S. 2).

Eine weitere Unterscheidung muss getroffen werden zwischen Normen mit allgemeinem und solchen mit kontext- bzw. akteursspezifischem Geltungsanspruch, z. B. rechtliche Vorgaben oder soziale Normen, die nur mit Blick auf einzelne Kontexte und Handelnde (etwa Angehörige einer ethnischen Gruppierung oder Religion) gelten (AA und IMSD 2017, S. 2). Da Friedensprozesse regelmäßig in einem Klima umstrittener Legitimität stattfinden, müssen sie in der Lage sein, eine kritische Masse sowohl an internationaler als auch lokaler Akzeptanz und Legitimität zu generieren, damit internationale Akteure und involvierte Nachbarstaaten, aber auch Konfliktakteure und betroffene Gesellschaften berechtigtes Vertrauen in diese Prozesse gewinnen können (Acharya 2004; Arnault 2014; Kraus ${ }^{8}$ 2011).

Ein Modell (in Anlehnung an Hellmüller et al. 2015; Abb. 1), das die genannten Kategorien zusammenführt, ordnet die für Mediation relevanten Normen nach ihrer jeweiligen Stellung im Mediationskontext an. Prozessbezogene Normen beziehen sich auf die Art und Weise der Verhandlung und damit darauf, wie während der Mediation (nicht) verhandelt werden sollte. Inhaltsbezogene Normen beziehen sich auf die Inhalte der Mediation und damit auf das, was während der Mediation (nicht)

\footnotetext{
8 Kraus, nach Namensänderung jetzt Holper.
} 
verhandelt werden kann. Quer dazu steht die Unterscheidung zwischen konsolidierten und nicht-konsolidierten Normen. Eine Norm gilt dann als konsolidiert, wenn von ihr nicht abgewichen werden kann, ohne dafür eine rechtfertigende Begründung abzugeben. Grundsätzlich kann angenommen werden, dass die Bindungswirkung von Normen mit dem Grad ihrer Konsolidierung steigt (AA und IMSD 2017, S. 3). Legt man die vier Kategorien (inhalts-/prozessbezogen, nicht-/konsolidiert) übereinander, ergeben sich vier allgemeine Typen von Normen, z.B. „prozessbezogen konsolidiert“. Ein besonderer Typ konsolidierter Normen ergibt sich aus der Definition von Mediation. Diese definitorischen Normen bestimmen Prozesseigenschaften, die erfüllt sein müssen, damit ein Mediationsprozess als solcher bezeichnet werden kann (Hellmüller et al. 2015, S. 2).

Im Ergebnis ist der Ordnungsrahmen der Friedensmediation als Handlungsfeld dadurch gekennzeichnet, dass er sich aus unterschiedlichen Normenarten mit unterschiedlichen Geltungsansprüchen und Bindungswirkungen zusammensetzt: neben Mediationsmethodik und -ethik, Völkerrecht und nationalem Recht, können für die Formulierung kontext- und akteursspezifischer Normen etwa werte-/ ideologiebasierte Elemente außenpolitischer Agenden, die internen Regularien internationaler Organisationen und die jeweiligen kulturell geprägten normativen Vorstellungen von Konfliktparteien Bedeutung entfalten. ${ }^{9}$

Es gibt dabei nur eine geringe Zahl konsolidierter Normen, die im jeweils relevanten Akteurskreis als allgemein gültig einzustufen sind: Zustimmung der Parteien (siehe 4.1.) dürfte beispielsweise nur unter Vertretern des fazilitativen Mediationsansatzes als konsolidiert betrachtet werden, unter denen eines machtbasierten Mediationsansatzes nicht. Letztlich sind nur Normen des Völkergewohnheitsrechts ${ }^{10}$ und quasi universell ratifizierte völkerrechtliche Verträge wie die UN-Charta oder die Genfer Konventionen von 1949 als quasi-konsolidiert zu betrachten, da deren universaler Geltungsanspruch weithin anerkannt ist, aber aus mancher wissenschaftlichen und politischen Sicht ebenfalls umstritten bleibt (Kolb 2015). Wie der Ordnungsrahmen im Einzelfall konkret aussieht, hängt folglich von der jeweiligen Parteien- und Drittparteienkonstellation und der Konfliktmaterie ab. Da diese Faktoren unendlich variierbar sind, gibt es (und kann es) keinen einheitlichen normativen Ordnungsrahmen für die Friedensmediation (geben). Nur der informierte und differenzierte Blick im Einzelfall erlaubt Orientierung, Wertschöpfung und Erkenntnis.

Nach dieser Übersicht über die für Friedensmediation relevanten Normenkategorien wird im Folgenden ein Blick auf ihre exemplarische Ausdifferenzierung durch die UN Guidance for Effective Mediation und die im Zuge ihrer Etablierung kritisierten Aspekte geworfen.

\footnotetext{
9 Eine nicht mediationsbezogene, gleichwohl aufschlussreiche grafische Aufschlüsselung unterschiedlicher Normdimensionen findet sich bei Wiener und Puetter (2009).

10 Völkergewohnheitsrecht basiert auf der Rechtsüberzeugung und der entsprechenden Praxis der Staaten, wobei Verhältnis von Überzeugung und Praxis zueinander und die Begrenzung auf Staaten umstritten sind (International Law Commission 2018).
} 


\section{Die UN Guidance for Effective Mediation: Ausdifferenzierung und Kritik methodisch-ethischer Normen}

Die im Jahr 2012 erschienene UN Guidance for Effective Mediation (Vereinte Nationen 2012) war und ist weiterhin Symbol und Katalysator der Professionalisierung der Friedensmediation (Wählisch 2016). Nach einem umfassenden konsultativen Prozess, der zahlreiche auf Mediation spezialisierte Organisationen sowie politische und akademische Akteure einbezog (MSN 2012), wurden in diesem Dokument bestehende methodisch-ethische Normen für die Gestaltung von Mediationsprozessen und Verweise auf relevante Bereiche rechtlicher Normen einheitlich zusammengefasst. Sie stellt damit mehr eine Erfassung und Konsolidierung derjenigen Normen dar, die bereits zuvor zur Anwendung kamen bzw. kommen sollten, als eine eigenständige Kodifizierung neuer Normen. Die besondere Relevanz der UN Guidance entstand durch den Umstand, dass sie überhaupt erstmals generische, einheitliche Richtwerte für professionelles Vorgehen in der Friedensmediation benannte und die Bedeutung dieser Normen für die Mediation explizit machte. Diese methodisch und ethisch begründete und rechtlich verortete Friedensmediation unterscheidet sich deutlich von dem, was etwa im Rahmen des Westfälischen Friedens als Mediation bezeichnet wurde und auch von dem, was noch bei der Abfassung der UNCharta der Vorstellung von Mediation entsprach. Dieses frühere völkerrechtliche Begriffsverständnis sah die Mediator*in als Drittpartei, die primär unverbindliche eigene Lösungsvorschläge unterbreitete, während nunmehr ihre Rolle als „Ermöglicher*in“ einer eigenständigen Konfliktlösung durch die Parteien im Vordergrund steht. Den Weg von dort zur UN Guidance bereiteten einige gescheiterte Kodifikationsunterfangen, die hinsichtlich der Mediation allgemein gehaltene Manila Declaration (Vereinte Nationen 1982) sowie das Handbook on the peaceful settlement of disputes between states (Vereinte Nationen 1992), welches jedoch lediglich eine deskriptive Bestandsaufnahme der Streitbeilegungspraxis darstellte (Würkert 2021). Daher ist die UN Guidance als das derzeit politisch und praktisch meist rezipierte und normativ umfassendste Dokument zu moderner Mediation in internationalen Friedensprozessen einzuordnen. Dies macht die UN Guidance zum natürlichen Ausgangspunkt einer Beschäftigung mit dem neu ausdifferenzierten Normengefüge in diesem Feld und mit den entsprechenden kritischen Gegenreaktionen (AA und IMSD 2017, S. 4).

\subsection{Die Normen der UN Guidance}

Die in der UN Guidance enthaltene Definition von Mediation führt aus, welche konstitutiven Voraussetzungen erfüllt sein müssen, damit ein Verfahren im Inneren als Mediation im Sinne der UN Guidance verstanden und nach Außen auch als solche bezeichnet werden kann. ${ }^{11}$

11 Das Mediation Support Network hat in einem Kommentar zur UN Guidance den Versuch unternommen, die Guidance auszulegen und zu definieren, welche Prinzipien beachtet werden müssen, damit die Verfahrensvoraussetzungen einer Mediation erfüllt sind (MSN 2012). 
Mediation is a process whereby a third party assists two or more parties, with their consent, to prevent, manage or resolve a conflict by helping them to develop mutually acceptable agreements (Vereinte Nationen 2012).

Aufbauend auf diese prozessorientierte Definition formuliert die UN Guidance acht Grundsätze (fundamentals) für die effektive Durchführung einer Friedensmediation:

- Preparedness

- Consent

- Impartiality

- Inclusivity

- National Ownership

- International Law and Normative Frameworks

- Coherence, Coordination and Complementarity

- Quality Peace Agreements

Der Grundsatz Preparedness nennt das umfassende Vorbereitetsein von Mediationsprozessen (mit Blick auf Wissen und Kompetenzen sowie politische, finanzielle und strukturelle Ressourcen der Mediator*in, des Mediationsteams und weiterer involvierter Akteure) als erste Voraussetzung verantwortungsvoller und glaubwürdiger Interventionen. Das Prinzip Consent bezeichnet die Zustimmung der Konfliktparteien zur Durchführung des Mediationsprozesses sowie zu Rahmen und beabsichtigten Formaten als zweite Bedingung für effektives Vermitteln. Impartiality beschreibt die erforderliche Unparteilichkeit der Vermittelnden, die jedoch ausdrücklich nicht mit Neutralität gleichgesetzt wird. Der Grundsatz Inclusivity übersetzt die Ziele der Partizipation und Repräsentation konfliktspezifisch relevanter Akteursgruppen auf die Logik von Mediationsverfahren und weist Schnittmengen zum direkt darauffolgenden Grundsatz, National Ownership, auf. Dieser bezieht sich zum einen auf die Aneignung des Prozesses, ausgehandelter Vereinbarungen und deren Umsetzung durch die Interessensgruppen im Konfliktland, zum anderen fragt er nach relevanten akteursspezifischen Normensystemen. International Law and Normative Frameworks benennt die Relevanz völkerrechtlicher und anderer Normen in der Mediation; die Integration der prozess- und materiell-rechtlichen Elemente an dieser Stelle kann als Beleg dafür gesehen werden, dass Friedensmediation zunehmend explizit im (Völker-)Rechtssystem verortet wird. Zudem sind Mediator*innen explizit angehalten, ihre handlungsleitenden Normen gegenüber den Konfliktparteien transparent darzulegen. Coherence, Coordination und Complementarity nennt Leitlinien zum verbesserten Austausch zwischen Mediationsakteuren; in zahlreichen Ländern und Organisationen wurde das Prinzip als Aufforderung zur Ausdifferenzierung von Mediation Support Strukturen und Kooperationsnetzwerken verstanden. Das Prinzip Quality Peace Agreements bezieht sich auf Inhalt und Umsetzung mediierter Vereinbarungen, muss also in der Dimension von Normen als Ergebnis der Friedensmediation verortet werden (IMSD 2017, S. 5; Vereinte Nationen 2012). 


\subsection{Normative Natur und Bindungswirkung}

Die oft gleichzeitig methodisch-technisch und ethisch ausgerichteten Grundsätze der UN Guidance beruhen auf in der Praxis geteilten Grundverständnissen und Erfahrungswerten von effektivem und gerechtem Vorgehen in der Friedensmediation. Ihre Formulierung bewegt sich an der Grenze zwischen Anleitung zur praktischen Ausgestaltung von Friedensmediationen und Best Practice-Empfehlungen aus dem Bereich Mediation Support. ${ }^{12}$ Die UN Guidance, teilweise als ,prozessuales soft law" der Friedensmediation beschrieben, hat keine rechtliche Bindungswirkung. Allerdings besteht für UN-Mediator*innen naturgemäß eine organisationsethische Bindung. Ebenso orientieren sich einige andere Organisationen in ihren Leitlinien, etwa die OSZE, eng an der UN Guidance, deren Inhalte sie weiter konkretisiert und in ihren normativen Ordnungsrahmen aufgenommen haben (OSCE 2014; AA und IMSD 2017, S. 4).

Trotz des Bekenntnisses der UN zu den in der Guidance formulierten Grundsätzen hat deren Missachtung in der Praxis auch für UN Mediator*innen regelmäßig keine relevanten Folgen - für staatliche und nicht-staatliche Mediator*innen ohnehin nicht. Ein sichtbares Zuwiderhandeln löst jedoch einen Rechtfertigungsbedarf in manchen Kreisen der professional community aus. Denn wenn definitorische Prinzipien wie Consent oder Ownership der Parteien ignoriert werden, bedeutet dies in der Konsequenz, dass das praktizierte Verfahren eben keine (gemäß dem ,state of the art“ durchgeführte) Mediation, sondern ein anderes diplomatisches Instrument darstellt, z. B. high-powered diplomacy oder arbitration (Kirchhoff 2008). Mediation im Sinne der UN Guidance unterstützt die Parteien bei ihrer eigenen Konfliktbearbeitung, die vermittelnde Drittpartei fällt selbst keine Entscheidungen über die Konfliktinhalte. Allerdings kann es für eine Verfahrensanpassung durchaus gute Gründe geben (Svensson 2007), etwa der klare Beweis, dass es ohne Einsatz von politischer Macht oder militärischem Eingreifen zu weiterer Eskalation kommen wird oder dass eine klare Entscheidung durch eine höhere Instanz notwendig ist (AA und IMSD 2017, S. 4).

Werden jedoch in mediationsgeeigneten Konstellationen die über Jahrzehnte zu mediationsspezifischen Verfahrensnormen gewonnenen Erfahrungswerte ${ }^{13}$ missachtet, so unsere Einschätzung, wird das Potenzial moderner Friedensmediationsmetho-

\footnotetext{
${ }^{12}$ Unter Mediation Support verstehen wir ,,activities that assist and improve mediation practices, e.g. training activities, developing guidance, carrying out research, working on policy issues, offering consultation, backstopping ongoing mediation processes, networking and engaging with parties" (Mediation Support Network, zuletzt besucht am 28.10.2019 https://mediationsupportnet.ethz.ch).

13 Ban Ki-Moon: „While all disputes and conflicts are unique and require specific approaches, there are good practices that should inform the approaches of all mediators" (Vereinte Nationen 2012, S. 1). Derartige Erfahrungswerte bewegen sich nach dem Cynefin Framework zwischen good practice und emergent patterns, da Interventionen in Konfliktsysteme nur wenige generalisierbare Ursache-Wirkungsbeziehungen aufweisen (Mason 2016). So haben empirische Studien sowohl Belege dafür gefunden, dass sich die Inklusion aller Konfliktparteien nicht positiv auf die Nachhaltigkeit des Friedens auswirkt, als auch dafür, dass die Beteiligung von Frauen und zivilgesellschaftlichen Akteuren (Nilsson 2012) sehr wohl einen positiven Effekt auf die Nachhaltigkeit eines ausgehandelten Friedens hat (Nilsson 2008, 2012; Paffenholz 2018). (Nilsson 2008) Zum Einfluss von Strafverfolgung auf Friedensprozesse ergibt sich ein ebenso komplexes empirisches Bild (Dancy und Wiebelhaus-Brahm 2017).
} 
dik und die darauf basierende Legitimierung des Verfahrens grundlos verschenkt. Dies gilt besonders dann, wenn ein Prozess explizit als Mediation bezeichnet wird und Konfliktparteien und Akteure der internationalen Gemeinschaft den Prozess einzig aufgrund der damit assoziierten oder ihnen zugesicherten Chancen unterstützen und ihm einen entsprechenden Vertrauensvorschuss gewähren. So wurde etwa im Rahmen der Genf II Gespräche zum Syrien-Konflikt aufmerksam analysiert, zu welchem Grad die in Aussicht gestellte zivilgesellschaftliche Partizipation umgesetzt wurde (Hellmüller und Zahar 2019). Die „guten Gründe“, die Kompromisse mit Blick auf die Beachtung von Verfahrensnormen nahelegen können, etwa dass ein nicht-konsensbasiertes oder nicht-ownership-basiertes Vorgehen angesichts der Druckkulisse von Zeitmangel und Gewalt kurzfristig erfolgsversprechender erscheint, sollten also intensiv auf ihre konkrete Stichhaltigkeit geprüft werden (AA und IMSD 2017, S. 4f).

\subsection{Rezeption und Kritik}

Die UN Guidance steht exemplarisch für einen historischen Paradigmenwechsel: Mit der Sammlung, Generalisierung und normativen Einfassung spezialisierten Erfahrungswissens hat sie maßgeblich die Professionalisierung eines Feldes vorangetrieben, das über Jahrhunderte in diplomatischen Traditionen und individuellen, nichtübertragbaren Fähigkeiten von Vermittler*innen verankert war (Convergne 2016). Die vergleichsweise hohe präskriptive Ausdifferenzierung der Guidance führte in dem gewohnheitsmäßig auf Flexibilität (,,whatever works") setzenden Feld der Friedensvermittlung erstmals eine Art Verfahrenskodex für ,gute“ Mediationsprozesse ein (Convergne 2016). Die damit - wie bei der Herausbildung einer jeden Profession - markierten Grenzen akzeptablen Handelns (Barnett und Finnemore 2004) geben Friedensmediator*innen Orientierung, Autorität, Legitimität und eine Berufsidentität, schränken aber auch die Manövrierfähigkeit ein, z.B. mit Blick auf Amnestien (Hellmüller et al. 2015).

In Praxis und Wissenschaft dürfte die UN Guidance anfangs vor allem deswegen so gut angenommen worden sein, weil sie einen bis dato fehlenden, erfahrungsbasierten Bezugsrahmen für Qualitätskontrolle und Evaluation lieferte (Hellmüller et al. 2015) und mit dezidiert unterstützender (statt regulierender oder rechenschaftsfordernder) Haltung an Vermittler*innen herantritt. Auch wenn sie als wesentlicher abstrakter Bezugsrahmen anerkannt und genutzt wird, wird die UN Guidance in ihrem konkreten praktischen Orientierungswert als eher gering eingeschätzt. Der zentrale Vorbehalt richtet sich auf die Generalisierbarkeit bzw. Rekontextualisierbarkeit ihrer Grundsätze (fundamentals). So wird immer wieder bezweifelt, inwieweit kontextgebundenes Best Practice-Wissen angesichts der Verschiedenartigkeit von Konfliktkontexten und Mediationsmethoden in der Praxis überhaupt auf andere Kontexte übertragbar ist (Mancini und Vericat 2016). Andere kritisieren, dass die Guidance zu breit und abstrakt formuliert sei, um praktisch anwendbar zu sein, ihr also noch zu viele Schritte zur echten Operationalisierung fehlen (Convergne 2016). Weitere Kritik richtet sich auf die nicht erkennbare Priorisierung der Grundsätze bzw. auf die fehlenden Abwägungskriterien für eine situative Priorisierung (Palmiano Federer 2016). 
Auf der Suche nach Gründen für ihren umstrittenen Stand fällt zunächst auf, dass die acht Grundsätze in ihrer relativen Abstraktheit in vielen Kulturräumen weitestgehend konsensfähig sein dürften. ${ }^{14}$ In dieser oder ähnlicher Form waren sie auch vor Veröffentlichung der UN Guidance schon Grundprinzipien von Mediation, sie bieten also bei strenger Sicht, ,wenig Neues“. Dass Mediationsverfahren im Kontext gewaltsamer Konflikte beispielsweise der Vorbereitung bedürfen, dürfte genau so wenig als ernst zu nehmende Einschränkung oder auch nur Neuerung wahrgenommen werden wie der Umstand, dass Konfliktbearbeitung die Einbeziehung relevanter Akteure erfordert.

$\mathrm{Zu}$ Verunsicherung führen die Grundsätze dennoch, weil hinter ihnen deutlich konkretere normative Forderungen gesehen oder vermutet werden als sie explizit zum Ausdruck bringen. Dabei geht es um Uneindeutigkeit in zentralen Fragen, im Fall von inclusivity etwa welche Akteure (Frauen, Zivilgesellschaft) tatsächlich so relevant sind, dass sie (ohne verbleibende Ermessensspielräume) inkludiert werden müssen und welcher implizite Referenzrahmen erforderlicher Inklusivität dem unterliegt. Weitere grundsätzliche Kritik richtet sich darauf, dass das liberale Werteparadigma, welches das Mediationsverständnis der UN und dessen Grundsätze durchdringt, als normative Setzung unreflektiert bleibt, ebenso wie die daraus resultierenden Legitimitäts- und Kontextualisierungsprobleme in der Praxis (Kraus 2011; Zeller und Schellekens 2017). So bleibt offen, wie die häufig aus transnational privilegierten Schichten stammenden Mediationsakteure in nicht-westlichen Konfliktkontexten nach liberal geprägten UN Grundsätzen verfahren sollen, wenn etwa politische und praktische Realitäten oder soziale und institutionelle Normen im Konfliktkontext diesen normativen Ansätzen entgegengerichtet sind. Dass diese Problematik die eigenen Prinzipien von ownership und inclusivity ad absurdum führt, hat im Peacebuilding Sektor bereits zu einem adaptive turn geführt (de Coning 2018), der inzwischen auch das Mediationsfeld erreicht.

Insgesamt lässt sich die UN Guidance als ein halb geglückter Versuch einstufen, durch Zusammenführung bestehender normativer Bausteine für deren Konsolidierung zu sorgen. Gelungen ist dies in der fazilitativen Mediation: dort kommt den definitorischen Normen der UN Guidance (Vereinte Nationen 2012) inzwischen ein weitgehend konsolidierter Status zu. Mit Blick auf das ganze Feld der Friedensmediation wirkt ihr Normierungsimpuls jedoch eher indirekt als Bezugspunkt für die normative Weiterentwicklung. Für die normenbejahenden Akteure ist die Guidance Anreiz zur präzisierenden Kritik und Verbesserung, für die normenskeptischen Akteure Bezugspunkt von Verwirrung und Abgrenzung. Entsprechend gegenläufig sind die systemischen Fortwirkungen: zum einen geglückte Präzisierungen und Übersetzungen in die mediationsmethodische Praxis durch andere, aktiv mit der Guidance arbeitende Akteure. Bespielhaft dafür ist der Reference Guide der OSZE zu Mediation and Dialogue Facilitation (OSCE2014). Zum anderen führt die Guidance zu starken normenkritischen bis normaversen Gegenreaktionen, wie sie in der pauscha-

\footnotetext{
14 Im Vergleich weisen beispielsweise die Mediationsstandards der Afrikanischen Union weitgehende Überlappungen mit der UN Guidance auf, sie enthalten u. a. mit gleicher Begrifflichkeit ownership, inclusivity und impartiality, andere Prinzipien wie consent und coherence, coordination and complementarity in eigener Begrifflichkeit wie non-threatening nature und linkages (ACCORD und African Union 2014).
} 
len Infragestellung des praktischen Nutzens von Normen für die Friedensmediation zum Ausdruck kommen:

A quick comparison of peace processes over the past 25 years shows no evidence that the absence of amnesty, the existence or absence of provisions on truth and reconciliation, or the inclusion of civil society have made a difference in terms of the durability or sustainability of those peace processes. What past experience does bear out is the complexity of the choices each one of these issues involves. [...] However attractive it may be to fuse peacemaking requirements with all the other values the international community currently stands for, this temptation must be resisted, not embraced (Arnault 2014).

Eine systematische Auswertung des ganzen Spektrums normierungskritischer Stimmen aus Wissenschaft und Praxis steht noch aus. Im Prinzip scheint jedoch bislang schlichtweg das normative Wissen zu fehlen, um eine in jedem Fall notwendige Klärung vornehmen zu können: was genau Gegenstand der relevanten Normen ist, wie sich diese Normen auf Konfliktinhalte, Prozessabläufe und den Handlungsspielraum von Mediator*innen beziehen, und warum es sinnvoll sein könnte, diese Normen zu erfüllen oder nicht, inklusive der potenziellen Risiken ihrer Vernachlässigung. Im nächsten Abschnitt sollen zu diesem Zweck drei komplementäre rechtliche Referenzsysteme vorgestellt werden, die in unterschiedlichen Konfliktkonstellationen und -kontexten unterschiedliche Relevanz entfalten können: akteursspezifische Normen, Transitional Justice und die Rolle von Gerichten.

\section{Komplementäre rechtliche Referenzsysteme: Akteursspezifische Normen, Transitionial Justice, Rolle von Gerichten}

Sind die Gründe und Ziele, die im eigenen oder kollektiven Interesse für die Einhaltung geltender Normen sprechen, nicht vollständig bewusst oder überzeugend, erscheinen Normen leicht als bloße Beschränkung der eigenen Handlungsgründe und -ziele. Die Bereitschaft sie zu wahren, wird entsprechend gering ausgeprägt sein. Daher sind die Kernargumente, weswegen in der Friedensmediation trotz ihres Charakters als flexibler, außergerichtlicher Streitbeilegungsmechanismus relevante rechtliche Normen berücksichtigt werden sollten, wichtig. Im Kern handelt es sich um drei Argumente: Erstens, dass es sich dabei nicht um aufgezwungene Begrenzungen handelt, sondern regelmäßig um die eigenen Normen der betroffenen Akteure. Zweitens kann in rein machtbasierten Aushandlungen der Schutz von bestimmten Gruppen Schaden erleiden, wenn normative Mindeststandards ignoriert werden. Drittens kann durch das Ausblenden normativer Dimensionen die Umsetzbarkeit und Nachhaltigkeit entsprechend normativ defizitärer Vereinbarungen in Mitleidenschaft gezogen werden, wenn diese Gegenstand rechtlicher Auseinandersetzungen - etwa vor Gericht - werden. 


\subsection{Akteursspezifische Normen ${ }^{15}$}

Die bisherigen Ausführungen haben die für Friedensmediation relevanten Normen weitgehend unabhängig von den handelnden Akteuren behandelt. In allen Phasen einer Friedensmediation stellt sich jedoch die Frage, welche spezifischen Normen welche Akteure an welche Erwartungen und zu welchem Grad binden.

Grundsätzlich sind Staaten an alle durch sie ratifizierten Verträge gebunden, sowie an die Normen des Völkergewohnheitsrechts. Materielle Rechtsnormen aus der Sphäre einer Konfliktpartei können ebenfalls klare Grenzen des gemeinsam rechtsgültig Verhandelbaren setzen und damit Teil des für den Mediationsprozess relevanten Ordnungsrahmens werden. Hat sich etwa ein Staat im Rahmen eines völkerrechtlichen Vertrages zu einem stärkeren Schutz bestimmter Menschenrechte verpflichtet, darf er als Konfliktpartei in der Mediation und in der Friedensvereinbarung nicht unter dieses Schutzniveau zurückfallen, wenn er nicht gegen das Völkerrecht verstoßen möchte (AA und IMSD 2017, S. 8).

Als normativ herausfordernd mit Blick auf akteursspezifische rechtliche Voreinschränkungen kann sich die Mediation unter Einbindung nicht-staatlicher bewaffneter Akteure erweisen. Es besteht weitgehender Konsens, dass auch Gewaltakteure an die grundlegenden Normen des humanitären Völkerrechts, mithin die Haager und Genfer Konventionen, gebunden sind. Auch Normen des zwingenden Völkerrechts gelten für sie im Grundsatz. Zudem verpflichten sich einige nicht-staatliche Gewaltakteure einseitig zur Einhaltung bestimmter Normen, die damit ebenfalls Teil des mediationsrelevanten Ordnungssystems werden. Die Bindung nicht-staatlicher Gewaltakteure an menschenrechtliche und darüber hinausgehende völkerrechtliche Verpflichtungen ist eine umstrittene Fragestellung, allerdings zeigt die völkerrechtliche Entwicklung in Teilen deutlich in diese Richtung (Henckaerts and Wiesener 2020; AA und IMSD 2017, S. 8).

Bei einer Beteiligung von Nichtregierungsorganisationen (NROs) an Prozessen der Friedensmediation entweder in der Rolle von Mediator*innen oder Unterstützer*innen einzelner Konfliktparteien stellt sich die praktisch äußerst relevante Frage, inwieweit diese Akteure eigentlich an völkerrechtliche Normen gebunden sind - und wie diese in der Verbreitung und Anwendung von Normen agieren (Hellmüller et al. 2017). Inwieweit NROs aufgrund zunehmender Mitwirkungsrechte in der internationalen Arena ein partieller Völkerrechtssubjektstatus eingeräumt werden kann, ist nicht geklärt. Ähnlich wie bei nicht-staatlichen Gewaltakteuren ist es überzeugend, dass auch für sie der Mindeststandard des zwingenden Völkerrechts gelten muss. Darüber hinaus sind NROs zusätzlich immer an die nationalen Rechtsnormen des Staates gebunden, in dem sie ihren Sitz haben. In Person der Drittpartei werden mindestens prozessuale, oft zudem materielle Rechtsnormen in das Verfahren getragen (AA und IMSD 2017, S. 8).

15 Teile dieses Abschnitts wurden in wortwörtlicher oder leicht angepasster Form aus dem Fact Sheet „Normativer Bezugsrahmen und völkerrechtliche Grundlagen der Friedensmediation“ (AA und IMSD 2017) übernommen; sie sind jeweils mit Quellenangaben als direktes oder indirektes Zitat kenntlich gemacht. 
Abb. 2 Zusammenwirken verschiedener Akteure in der Friedensmediation. (In Anlehnung an Grafik in AA und IMSD 2017, S. 9, in Anlehnung an Leffmann, Keno. 2016. Der völkerrechtliche Ordnungsrahmen der Mediation in internationalen Friedensprozessen. Unveröffentlichte Masterarbeit. EuropaUniversität Viadrina Frankfurt [Oder])

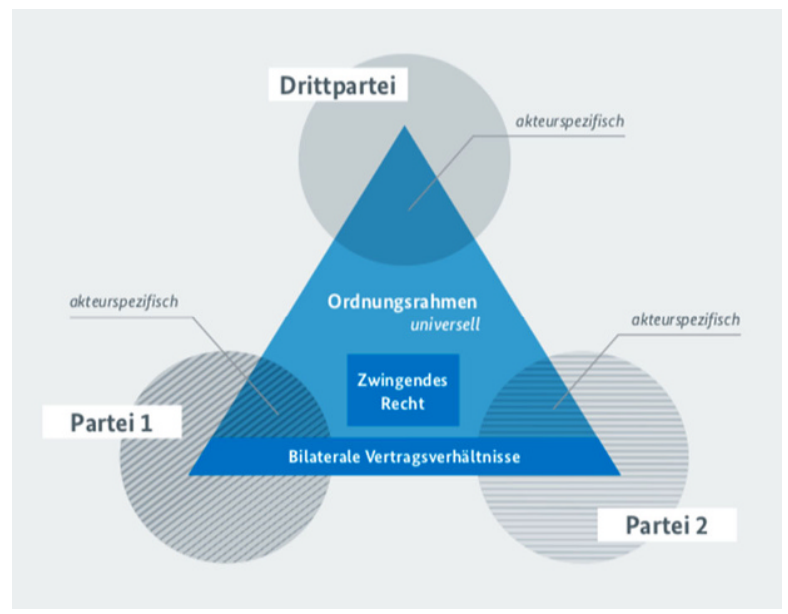

Für den rechtsnormativen Ordnungsrahmen einer Friedensmediation macht es also durchaus einen Unterschied, ob die Mediator*innen für die UN, EU oder eine sonstige zwischenstaatliche Organisation, für einen Staat oder eine NRO arbeiten. So agieren etwa UN-Mediator*innen im Rechtsrahmen der UN-Charta, der Resolutionen des Sicherheitsrates und der Generalversammlung sowie interner UNRegularien. Staatliche Mediator*innen hingegen sind neben der UN-Charta und den Resolutionen des Sicherheitsrats auch an das gesamte Paket zuvor beschriebener völkerrechtlicher Verpflichtungen gebunden und darüber hinaus an die jeweils anwendbaren Normen der nationalen Rechtsordnung (AA und IMSD 2017, S. 8).

Darüber hinaus sind Vorstellungen davon, was im Umgang mit Konflikten adäquat und gerecht ist, stets Ausdruck konkreter kultureller Prägungen der relevanten sozialen, institutionellen, politischen und religiösen Systeme (Reckwitz 2000). Diese kulturell geprägten normativen Vorstellungen können dazu führen, dass die verhandelnden Personen, die von ihnen vertretenen Gruppen und Gesellschaften sowie die Drittparteien jeweils sehr unterschiedliche Erwartungen, Wahrnehmungen und Bewertungen mit Blick auf Konfliktbearbeitungsverfahren und darin getroffene Vereinbarungen haben (Kraus 2011). Beispielsweise betonen manche Staaten selektiv stärker den Grundsatz der Nicht-Einmischung in staatliche Souveränität (u. a. in Zusammenhang mit Kritik an Menschenrechtsverletzungen). Ähnliches war kürzlich bei der Zurückhaltung Chinas als Mediator angesichts der von einer UN-Kommission geforderten Anklage zu Verbrechen an den Rohingya in Myanmar zu beobachten (Song 2019; Tisdall 2018). Dass historisch gewachsene kulturelle Normen oft nicht trennscharf von realpolitischen oder wirtschaftlichen Motiven zu unterscheiden sind und zur Rechtfertigung letzterer genutzt werden können, relativiert jedoch nicht die Signifikanz und Legitimität kultureller Prägungen von Normen per se. So gilt es in jedem Fall ein Bewusstsein dafür zu entwickeln, dass sich Akteure aus kollektiv verankerten kulturellen Gründen zu dem und innerhalb des internationalen Normensystem(s) unterschiedlich positionieren können und dies je nach Akteurskonstellation auch zu konfligierenden Situationen führen kann (AA und IMSD 2017, S. 8; Abb. 2). 
Das in der Grafik verdeutlichte Zusammenwirken dieser unterschiedlichen Akteure in der Friedensmediation findet nicht nur innerhalb des beschriebenen Ordnungsrahmens statt, sondern prägt diesen Ordnungsrahmen auch für zukünftige Friedensprozesse. Staatenpraxis und Rechtsüberzeugungen, die elementar für die Genese von Völkergewohnheitsrecht sind, manifestieren sich regelmäßig gerade in Konflikten und Vermittlungsprozessen. Die oben skizzierte Thematik etwa, ob und wie weit nicht-staatliche Gewaltakteure und NROs an Völkerrecht gebunden sind bzw. inwiefern Brüche $\mathrm{zu}$ Konsequenzen führen, ist etwa eine Frage, die sich derzeit in diesem Umfeld entscheidet; Vermittler*innen agieren in dieser Hinsicht auch als aktive Weiterentwickler*innen von Normen (Hellmüller et al. 2017). Auch die durch Sicherheitsratsresolution 1325 angestoßenen Entwicklungen im Bereich des Schutzes von Frauen im bewaffneten Konflikt und bezüglich ihrer Beteiligung im Friedensprozess kann hier verortet werden (AA und IMSD 2017, S. 9).

Schließlich muss in jedem konkreten Einzelfall analysiert werden, welcher Akteur durch welche Normen zu welchem Grad gebunden ist. Nicht nur die Konfliktparteien, sondern gerade auch die Drittpartei selbst sind dieser Analyse zu unterziehen. Die Wahl der Drittpartei bzw. die hinter ihr stehende Organisation oder Mandatgeber*in haben oftmals einen nicht zu unterschätzenden Einfluss auf die normative Konstellation. Dies kann insbesondere im frühen Stadium des Prozessdesigns relevant werden. Während die Konfliktakteure für die Bewertung ihres spezifischen eigenen Normgerüsts selbst verantwortlich sind, ist es gleichwohl bis zu einem gewissen Grad Aufgabe der Drittpartei sicherzustellen, dass alle anderen beteiligten Akteure informiert handeln können, d.h. auch in Kenntnis etwaiger rechtlicher Vorbedingungen auf Seiten aller Akteure (Breidenbach 2005).

\subsection{Transitional Justice ${ }^{16}$}

Rechtliche Normen begrenzen nicht nur die Gestalt des Friedensprozesses, sondern sind selbst gleichzeitig Mittel und Gegenstand der gesellschaftlichen Gestaltung, die während und nach einem Friedensprozess stattfindet. Diese transformative Funktion des Rechts soll der Begriff „Transitional Justice“ erfassen (AA und IMSD 2017, S. 10).

Transitional Justice gehört zu den zentralen Konzepten der Friedenskonsolidierung in Nachkriegsgesellschaften, die seit Mitte der 1990er Jahre von der internationalen Gemeinschaft und der globalen Zivilgesellschaft entwickelt wurden, um nachhaltigen Frieden in Postkonfliktgesellschaften zu gewährleisten (Kirchhoff 2009). Der Begriff umfasst sämtliche Maßnahmen, mit denen (Menschen-)Rechtsverletzungen und Gewalterfahrungen geahndet und gesellschaftlich bearbeitet werden sollen. Dies kann sich auf internationale oder nationale strafrechtliche Verfahren, aber auch auf außergerichtliche Versöhnungsstrategien von Tätern und Opfern eines Konflikts, Reparationsleistungen (Rückerstattungen, Entschädigungen), auf die Demobilisierung von Tätern oder auf die Wahrheits- und Faktenfindung beziehen. Die für das

16 Teile dieses Abschnitts wurden in wortwörtlicher oder leicht angepasster Form aus dem Fact Sheet „Normativer Bezugsrahmen und völkerrechtliche Grundlagen der Friedensmediation“ (AA und IMSD 2017) übernommen; sie sind jeweils mit Zitatnachweisen kenntlich gemacht. 
Feld Transitional Justice geltenden Normen prägen Friedensverhandlungen zunehmend. Immer häufiger und sichtbarer legen Friedensvereinbarungen die Eckpunkte für deren Umsetzung fest (European Union External Action Service 2012). Ziel vieler Friedensprozesse und damit der in ihrem Rahmen stattfindenden Friedensmediationen ist die Etablierung stabiler Gesellschaften, getragen durch ein Mindestmaß an Rechtssicherheit und Rechtsstaatlichkeit. Recht als gesellschaftliches Lenkungsund Gestaltungsinstrument muss sich nach dem in der Regel erfolgten Teilzusammenbruch der bisherigen rechtlichen Ordnung seine Legitimität darin jeweils neu erarbeiten (AA und IMSD 2017, S. 10).

Transitional Justice wird dabei regelmäßig auf den Zielkonflikt „Frieden oder Recht“" (peace versus justice) reduziert, doch bei genauem Hinsehen stehen die Ziele nicht in einem Konkurrenz-, sondern in einem Interdependenzverhältnis: ohne ein Ende der Gewalt (Frieden) könnten Individuen auch nicht von Aufarbeitung, Entschädigung und Menschenrechten (Recht) profitieren und umgekehrt. Transitional Justice muss in diesem Kontext beispielsweise mit normativ komplexen Phänomenen wie formal rechtsgültigem (staatlichem) Unrecht oder der Frage nach Entschädigungsansprüchen individueller Opfer umgehen (AA und IMSD 2017, S. 10). Dass Interdependenz dabei nicht mit spannnungsfreier Komplementarität zu verwechseln ist, verdeutlichen die Beispiele zur Rolle von Gerichten im nachfolgenden Abschnitt (4.3).

Die zentralen normativen Prinzipien im Bereich Transitional Justice basieren auf den Grundsätzen, die Louis Joinet für die UNO-Menschenrechtskommission entwickelte. Ziel war die Bekämpfung der Straflosigkeit bei schweren Menschenrechtsverletzungen und Verstößen gegen das humanitäre Völkerrecht. Die vier Schlüsselbereiche sind das Recht auf Gerechtigkeit, das Recht auf Wiedergutmachung, das Recht auf Wahrheit und die Garantie der Nichtwiederholung. Diese vier Prinzipien erkennen die Rechte der Opfer an und legen die Pflichten des Staats fest (Joinet 1997). Die UN und die EU (Council of the European Union 2009) streben eine Berücksichtigung der sich immer stärker ausdifferenzierenden Transitional Justice Prinzipien innerhalb von durch sie durchgeführten oder unterstützten Friedensvermittlungen ausdrücklich an. Andere Praxisakteure betrachten dies als normative Überfrachtung des Mediationsverfahrens und beklagen einen weiteren Verlust an inhaltlichem Spielraum und Ergebnisflexibilität der Vermittler*in. Für die Praxis der Friedensmediation gilt es daher, künstliche Gegensätze zu vermeiden, wofür sich der komplementäre Ansatz „Frieden im Recht/Recht im Frieden“ anbietet. Transitional Justice stellt damit einen weiteren entscheidenden Schritt auf dem Weg zu einem ganzheitlichen Konfliktlösungsansatz dar, der nicht starre Dichotomien in den Vordergrund rückt, sondern einen methodisch professionellen Umgang mit Normkonflikten ermöglicht (AA und IMSD 2017, S. 10). 


\subsection{Die Rolle von Gerichten ${ }^{17}$}

Dieselben Konflikte, die in Friedensmediationen verhandelt werden, können auch in internationalen, hybriden, regionalen und nationalen Gerichtshöfen verhandelt werden. Entsprechend können dort rechtskräftig vollstreckbare Urteile über (Teil-)Inhalte zukünftiger oder bereits bestehender Friedensvereinbarungen gefällt, Elemente für rechtswidrig erklärt oder entsprechende Klagen angestrengt werden. Wie das Zusammenspiel mit den verschiedenen Normen und Gerichten im Einzelfall genau abläuft, ist dabei ebenso komplex wie maßgeblich für die Durchführung normativ abgesicherter Friedensmediationen. Allein die Tatsache, dass Friedensverträge nachträglich gerichtlich überprüft und evtl. für partiell rechtswidrig erklärt werden können, sollte grundsätzlich den Aushandlungsprozess zwischen Parteien und Mediator*in prägen. Eine Voraussetzung dafür ist präzises, belastbares Wissen der Drittpartei über die normative Anatomie der Situation (AA und IMSD 2017, S. 12).

Mit Blick auf das Strafrecht kommt dem Internationalen Strafgerichtshof (IStGH) die größte Bedeutung zu. Bei international strafrechtlich relevanten Vorkommnissen prüft die Chefankläger*in des IStGH nach Art. 15 des Rom-Statuts, ob Anlass zur Einleitung eines Ermittlungsverfahrens besteht, welches letztendlich zum Erlass von Haftbefehlen führen kann. Grundsätzlich ist der IStGH allerdings während eines Friedensprozesses nicht zuständig, solange der betroffene Staat willens und in der Lage ist, selbst ernsthaft Ermittlungen und Strafverfolgung durchzuführen. Die Vorermittlungen und die damit einhergehenden Berichte des betroffenen Staates haben auch den Zweck, diese Bereitschaft und Fähigkeit zu überprüfen (AA und IMSD 2017, S. 11).

Ein anschauliches Beispiel für die Relevanz des internationalen Strafrechts ist etwa der kolumbianische Friedensprozess, den der IStGH im Rahmen von Vorermittlungen eng begleitet hat. In Kolumbien hat diese Rolle von Recht und Gerichtsbarkeit dazu beigetragen, die Unausweichlichkeit der Strafverfolgung für schwerste Verbrechen im Friedensprozess präsent zu halten. Damit hat der IStGH dafür Sorge getragen, dass in Kolumbien ein eigenständiger Mechanismus zur Konfliktaufarbeitung entsteht, der eine strafrechtliche Verfolgung miteinschließt. Einerseits zeigt das Beispiel Kolumbiens dabei den positiven Einfluss einer unabhängigen internationalen Strafverfolgung als feste Größe innerhalb eines Friedensprozesses (Urueña 2017). Andererseits zeigt er, dass an sensiblen Stellen solcher Prozesse Bedarf nach Zurückhaltung bei normativer Einflussnahme - etwa ein Verschieben normativ einschlägiger Sanktionen - bestehen kann. Ein Mittel hierfür ist der Aufschub von Ermittlungen oder Strafverfolgung nach Art. 16 des Rom-Statuts, der allerdings einer Kapitel-VII-Resolution durch den Sicherheitsrat bedarf. Angesichts dieser hohen Hürde stellt sich Art. 53 Abs. 1 c) u. Abs. 2 c) des Rom-Statuts als vielversprechender dar, nach dem die Chefankläger*in von Ermittlungen oder Strafverfolgung absehen kann, sofern diese „,nicht im Interesse der Gerechtigkeit lieg[en]“ (Rodman 2009; de Souza Dias 2017). Auch hier liegt die Einschätzung jedoch einzig bei

\footnotetext{
17 Teile dieses Abschnitts wurden in wortwörtlicher oder leicht angepasster Form aus dem Fact Sheet „Normativer Bezugsrahmen und völkerrechtliche Grundlagen der Friedensmediation“ (AA und IMSD 2017) übernommen; sie sind jeweils mit Zitatnachweisen kenntlich gemacht.
} 
dem*der Chefankläger*in und lässt sich nicht durch Mediationsakteure beeinflussen (AA und IMSD2017, S. 11).

Ein kontrovers diskutiertes Beispiel für das Spannungsfeld zwischen methodischen- und rechtlichen Normen sowie strategischen Imperativen der Friedensmediation sind die vom Internationalen Strafgerichtshof (IStGH) erlassenen Haftbefehle gegen den damaligen sudanesischen Präsidenten Omar al-Bashir und führende Mitglieder der ugandischen Rebellengruppe Lord Resistance Army (LRA). Der 2005 gegen führende Mitglieder der LRA erlassene Haftbefehl schränkte die Verhandlungsbereitschaft der LRA ein, da deren Führung auf Straffreiheit beharrte. Die ugandische Regierung, die den Konflikt mit der LRA zuvor an den IStGH überwiesen hatte, bereute dem Anschein nach diese Entscheidung später (Mediation Support Network 2013; Piiparinen 2013). Wie auch im Fall des 2009 und erneut 2010 erlassenen Haftbefehls gegen Omar alBashir zeigt sich der Normenkonflikt in der Gestalt, dass die Haftbefehle mangels ausreichender Kooperation und Durchsetzungsmechanismen der Gerichte nicht zum Erfolg führten und zugleich die Bereitschaft der damit konfrontierten Verhandlungsparteien zur friedlichen Beilegung des Konflikts gefährdeten (Rodman 2009) (IMSD 2017).

Im Bereich des Strafrechts sind darüber hinaus oft hybride Strafgerichte mit der Aufarbeitung der in Friedensmediationen verhandelten Konflikte befasst. Ihr Hybridcharakter rührt daher, dass sie einerseits auf einem völkerrechtlichen, andererseits auf einem nationalen Rechtsakt basieren. Sie werden oftmals nach innerstaatlichen Konflikten oder nach innerstaatlich begangenen Verbrechen in Kooperation mit dem jeweiligen Staat gegründet. Beispiele sind etwa das Rote-Khmer-Tribunal in Kambodscha, die Serious Crimes Panels in Osttimor oder das Sondertribunal für den Libanon (AA und IMSD2017, S. 11).

Die Bedeutung von hybriden Strafgerichten erschließt sich besonders gut am Beispiel des Sondergerichtshofs für Sierra Leone. Dieser erklärte, die zuvor im Friedensvertrag von Lomé enthaltene Amnestieklausel sei kein Hindernis für die Strafverfolgung. Ein Friedensvertrag innerhalb eines internen bewaffneten Konflikts sei kein völkerrechtlicher Vertrag und könne somit ein hybrides Strafgericht, das außerhalb der nationalen Rechtsordnung stehe, nicht binden. Vielmehr sei eine Amnestie für schwerste Verbrechen ein Verstoß gegen das Völkerrecht ${ }^{18}$ (AA und IMSD 2017, S. 11)

Der normative Ordnungsrahmen von Friedensmediation wird zudem, wie oben erwähnt, regelmäßig durch Menschenrechtsnormen und die Rechtsprechung regionaler Menschenrechtsgerichtshöfe beeinflusst. So hat der Interamerikanische Gerichtshof für Menschenrechte schon früh Blankett-Amnestien als unvereinbar mit den Rechten der Opfer und ggf. ihrer Hinterbliebenen erklärt und damit erst die strafrechtliche Aufarbeitung lateinamerikanischer Diktaturen ermöglicht (Binder 2011). Diese umfangreiche Rechtsprechung hat auch den Friedensprozess in Kolumbien intensiv

18 Prosecutor v. Kallon, Case No. SCSL-2004-15-AR72(E), Decision on Challenge to Jurisdiction: Lomé Accord Amnesty (Mar. 13, 2004). 
geprägt und wurde von allen Parteien im Detail zur Kenntnis genommen. Jenseits von Amnestien zeigt der Fall Sejdić und Finci des Europäischen Gerichtshofs für Menschenrechte (EGMR), wie weit die gerichtliche Überprüfbarkeit und rechtliche Aushebelbarkeit von Friedensverträgen gehen kann und wie umfassend die Mediationspraxis die normative Dimension ihrer Ergebnisse aus diesem Grund absichern sollte. Der EGMR hatte in diesem Fall bestimmte Verfassungsnormen BosnienHerzegowinas, welche das Ergebnis des Friedensvertrages von Dayton von 1995 gewesen waren, für rechtswidrig erklärt ${ }^{19}$ (AA und IMSD 2017, S. 11).

Auch die Rolle nationaler Rechtsnormen und Gerichte im normativen Gefüge moderner Friedensmediation kann schließlich kaum überschätzt werden. Nationale Gerichte prägen Aufarbeitungsprozesse in ihrem Land und können die Grundlagen eines Friedens- oder Übergangsprozesses ebenfalls rechtlich überprüfen. So hat etwa das südafrikanische Verfassungsgericht die Regelung zu innerhalb der Wahrheits- und Versöhnungskommission erlassenen Amnestien für verfassungskonform erklärt. ${ }^{20}$ Das deutsche Bundesverfassungsgericht wiederum bestätigte die vorhergehende Rechtsprechung im Fall der Mauerschützen, die den Verweis auf die Rechtmäßigkeit des Schießbefehls nach dem Recht der DDR nicht anerkannt hatte ${ }^{21}$ (AA und IMSD 2017, S. 12).

Nach der Zusammenschau von drei rechtlichen Bezugsrahmen, die den kontextabhängigen normativen Ordnungsrahmen von Friedensmediationen prägen, wird im letzten Schritt anhand von konkreten Beispielen aufgezeigt, wie kollidierende Normen systematisch miteinander abgewogen werden können.

\section{Abwägung von Normen in der Mediationspraxis}

Eine vollständige Erfüllung aller in einem Friedensprozess relevanten Normen ist weder möglich noch nötig. Es ist jedoch machbar und notwendig, eine differenzierte priorisierende Abwägung zwischen normativem Anspruch, Machbarkeit und Tragbarkeit vorzunehmen, also auszuloten, wie die jeweils relevantesten Normen in der konkreten Situation einzeln und zusammen bestmöglich berücksichtigt werden können und sollten.

\subsection{Ausloten der Grenzen und Spielräume des situationsspezifischen normativen Rahmens}

Im Mediations-Alltag wird das Ziel dieser Abwägung oft eine pragmatische Sondierung sein, wie die Normen bzw. deren Absichten (im Prinzip: Rechtsgültigkeit/

\footnotetext{
19 Konkret handelte es sich um Vorschriften, die für politische Posten einen ethnischen Proporz vorsahen und damit Minderheiten gänzlich ausschlossen (SEJDÍC \& FINCI V. BOSNIA AND HERZEGOVINA . App. Nos. 27996/06 \& 34836/06. At http://www.echr.coe.int. European Court of Human Rights (Grand Chamber), December 22, 2009.).

20 Azanian Peoples Organisation (AZAPO) and Others v President of the Republic of South Africa and others Case CCT 17/96.

21 BVerfG, Beschluss vom 24.10.1996, 2 BvR 1851, 1853, 1875 und 1852/94.
} 
Schutz des Schwächeren) im vorliegenden Kontext erfüllbar sind und welche normativen Abweichungen mit Blick auf die zu erwartenden Konsequenzen zumutbar und rechtfertigbar sind. Anspruch, Machbarkeit und Akzeptabilität lassen sich in im Dreischritt ideales Sollen, konkretes Können, kontextualisiertes Dürfen auf ein reales Sollen hin miteinander abwägen (AA und IMSD 2017, S. 12). ${ }^{22}$ Dabei werden mediationsmethodische, -ethische oder völkerrechtliche Normen (ideales Sollen) mit der im jeweiligen Konfliktkontext situativ bestehenden Handlungs- und Einflussfähigkeit der Mediator*in (konkretes Können) und der Akzeptabilität von potenziellen, durch normabweichendes Verhalten entstehenden Kosten (kontextualisiertes Dürfen) abgeglichen.

Eine solche Sondierung setzt voraus, dass bekannt ist, wo im konkreten Fall normative Grenzen und Spielräume liegen. Dafür ist zu klären, welche Normen einerseits eingehalten werden müssen, weil sie einen zwingenden Status haben oder weil die zu erwartenden negativen Auswirkungen im konkreten Fall nicht tragbar sind, und wo andererseits flexibel agiert werden darf, weil die negativen Auswirkungen im konkreten Fall gering oder hinnehmbar sind. Wichtig ist dabei, die Konsequenzen für wirklich alle potenziell von einer Entscheidung betroffenen Akteure zu prüfen: Die Konfliktbeteiligten, die Konfliktbetroffenen, die Gesellschaft, die Mediator*innen und ihre Auftraggeber*innen sowie evtl. die nationale und internationale Gemeinschaft (AA und IMSD 2017, S. 12).

Mit Blick auf methodisch-ethische Verfahrensgrundsätze bildet die echte (also auch nicht subtil erzwungene) Zustimmung der Parteien zu Prozess und Vereinbarung (Consent) eine normative Mindestvoraussetzung (ideales Sollen), wenn vermieden werden soll, dass die Konfliktparteien die Aushandlung und Umsetzung von Vereinbarungen torpedieren (AA und IMSD 2017, S. 12). Diese definitorische Norm ist implizit in der Definition von Mediation der UN Guidance enthalten (Vereinte Nationen 2012). Angesichts der beschränkten Möglichkeiten des Vertrauensaufbaus unter Zeitdruck (konkretes Können) kann z. B. im Einzelfall abgewogen werden, ob die Risiken einer nur inkrementellen oder sequenziellen Zustimmung und etwa ,nachhelfender“ Druckmittel tragbar sind (kontextualisiertes Dürfen).

Auf völkerrechtlicher Ebene werden in der Sondierung zahlreiche völkerrechtliche Normen relevant, die in zwingendes und sonstiges (einfaches) Recht zu unterteilen sind (ideales Sollen). Zwingendes Völkerrecht bezeichnet, wie oben beschrieben, die kleine, aber vermittlungspraktisch relevante Gruppe an Normen, deren Verbindlichkeit für sämtliche Akteure im Grundsatz nicht zur Disposition steht. Zwingendes Völkerrecht ist besonders tief im Rechtsbewusstsein verankert; in der Normenhierarchie stehen solche Vorschriften über sonstigem Vertrags- und Gewohnheitsrecht. Aus politischer und ethischer Sicht ist das zwingende Völkerrecht für den Zusammenhalt der Völkergemeinschaft zudem unabdingbar, da es als ein Mindeststandard die globale normative Diversität und Mächte-Multipolarität überbrückt. Das sonstige Völkerrecht umfasst Normen, die zwar durchaus universell anwendbar sein können, deren Verbindlichkeit jedoch durch nachfolgend entstandene, anderslautende völ-

\footnotetext{
${ }^{22}$ Der Dreischritt differenziert die rechtsphilosophische Abwägung zwischen idealem und realem Sollen (Sieckmann 2018) mit dem ethischen Grundsatz „Sollen impliziert Können“ und den kontextabhängigen Akzeptanzspielräumen von Normen.
} 
kerrechtliche Normen oder durch das Kündigen oder Aussetzen eines Vertrags aufgehoben werden kann (zumindest solange es sich nicht gleichzeitig um eine Norm des Völkergewohnheitsrechts handelt). Werden im Rahmen der Friedensmediation Rechtsnormen für die nationale Rechtsordnung geschaffen oder wird deren Schaffung vorgesehen, dürfen diese nationalen Normen jenen des Völkerrechts nicht entgegenstehen. Selbst Verfassungsnormen dürfen im Ergebnis nicht im Widerspruch zum Völkerrecht stehen (kontextualisiertes Dürfen). Wenn es in Friedensmediationen unmöglich erscheint, diese Vorgaben zu erfüllen (konkretes Können), liegt womöglich eine Normenkollision vor. Diese Konstellation wird im folgenden Abschnitt betrachtet.

\subsection{Umgang mit Normen-Kollisionen}

Bei Normen-Kollisionen, in denen verschiedene normative Imperative kollidieren oder sich sogar dilemmatisch ausschließen, ist in der Abwägung ein anderes Vorgehen notwendig. Solche Normenkonflikte lassen sich unterteilen in Kollisionen innerhalb derselben normativen Sphäre (etwa wenn internationale und nationale Rechtsnormen miteinander konkurrieren) und Kollisionen zwischen Normen aus unterschiedlichen Sphären (etwa wenn methodisch-ethische gegen rechtliche oder politische Prioritäten stehen) (AA und IMSD 2017, S. 13). In beiden Fällen geraten zwei oder mehr Normen, deren Handlungsimperative situativ nicht vereinbar und nicht hierarchisierbar sind bzw. erscheinen, miteinander in Konflikt - oft weil die Normen aus verschiedenen Normen(sub)systemen mit unterschiedlichen übergeordneten Zielsetzungen stammen (Kraus et al. 2019; Kraus 2011). So können zwischen dem mediationsmethodischen Ziel der Effektivität, dem mediationsethischen Ziel der Verfahrensgerechtigkeit und den rechtlichen Zielen der Rechtsgültigkeit und des Schutzes des Schwächeren grundlegende normative Zielkonflikte entstehen.

Bevor wir anhand von zwei Beispielen exemplarisch durchspielen, wie ein sinnvoller Umgang mit diesen Kollisionen aussehen kann, sollen drei klassische Methoden zum Umgang mit Normenkonflikten vorgestellt werden, mit denen kontextspezifisch konkurrierende Imperative ausbalanciert werden können: Priorisierung, Sequenzierung und Kompartmentalisierung (Kraus et al. 2019). Bei einer Priorisierung wird die Notwendigkeit der Beachtung von Normen abgestuft, sodass eine höchstpriorisierte Norm vollständig umgesetzt wird, eine zweitprioriserte teilweise und etwa eine drittpriorisierte nicht (Waldman 2011). Es kann auch in Form einer Sequenzierung vorgegangen werden, bei der nicht gleichzeitig umsetzbare Normen nacheinander in einer bestimmten Reihenfolge berücksichtigt werden (Weiss und Rosenberg 2003). Hier sind bereits verschiedene Formen etabliert: mit einer inkrementellen Sequenzierung (easy-to-hard), die zuerst die einfacher und danach die schwieriger realisierbaren Forderungen berücksichtigt, oder mit einer Agreementin-Principle Sequenzierung, die früh im Prozess eine übergeordnete Vereinbarung anstrebt (etwa durch Aufnahme des Punktes „Umgang mit Kriegsverbrechen“ auf die Agenda), die Aushandlung der Details aber erst zu einem späteren, strategisch günstigeren Zeitpunkt angeht. Weitere Methoden sind die abschichtende Sequenzierung (hard-to-easy), die die größte normative Hürde zuerst in Angriff nimmt, um kleinere Herausforderungen danach leichter bewältigen zu können, und die Komitee- 
Tab. 1 Fallbeispiel 1/Mediation Support für als ,terroristisch“ eingestufte Akteure. (In Anlehnung an Grafik in AA und IMSD 2017, S. 14)

\begin{tabular}{lll}
\hline $\begin{array}{l}\text { Frage im Zen- } \\
\text { trum der Nor- } \\
\text { menkollision }\end{array}$ & $\begin{array}{l}\text { Darf bzw. soll eine durch ein Außenministerium finanzierte Nichtregierungsor- } \\
\text { ganisation für Führungspersonen einer als ,terroristische“ Vereinigung gelisteten } \\
\text { Organisation Mediation Support (z. B. Verhandlungstraining) leisten, um diese für } \\
\text { Waffenstillstandsverhandlungen zu qualifizieren? }\end{array}$ \\
\hline \begin{tabular}{lll} 
Relevante & Pro: Mediationsmethodische und -ethische Prinzipien der Inklusivität, Ownership \\
Normen & $\begin{array}{l}\text { Contra: Strafrechtliche Normen zur Nichtunterstützung, ,terroristischer“ Vereinigun- } \\
\text { gen, ethisches Prinzip des Do No Harm }\end{array}$ & \\
\hline $\begin{array}{l}\text { Kosten-Nutzen- } \\
\text { Abwägung: }\end{array}$ & Pro: Inklusivität und Ownership & Contra: ,Antiterrorismusgesetze“ \\
$\begin{array}{l}\text { Beachtung vs. } \\
\text { Missachtung }\end{array}$ & $\begin{array}{l}\text { Steigerung (statt Stagnation) der Verhand- } \\
\text { lungskompetenz, Integration (statt weiterer }\end{array}$ & Und Do no harm \\
& Ausschluss) der Interessen einer Konfliktpar- & Strafkeit der Drittpartei
\end{tabular}
\end{tabular}

Ausschluss) der Interessen einer Konfliktpartei mit großer Störungsmacht, Minimierung (statt Risiko der Intensivierung) von Kampfhandlungen

Evtl. politische bzw. moralische Aufwertung des (statt Bestätigung eines Nicht-)Status der gelisteten Gruppe

Ausschluss (statt Risiko) einer Aufwertung der Gruppe und Instrumentalisierung der Mediationsarbeit durch die Gruppe

Annäherung an (statt Distanz zu) strafrechtlich relevantem Bereich für die Drittpartei

Ausschluss (statt Risiko) einer Stärkung auch der gewaltsamen Handlungsfähigkeit der Gruppe

Handlungsmöglichkeiten zur Ausbalancierung

\begin{abstract}
Beratung/Begleitung von Maßnahmen durch Rechtsexpert*innen und Regionalexpert*innen, um Vorwurf strafbarer Unterstützung sowie Risiken der Stärkung gewaltsamer Vorgehensweisen kontinuierlich entgegenzuwirken
\end{abstract}

Strafrechtlich potenziell sensible Maßnahmen (z. B. Geldzuwendungen) durch Kooperationspartner durchführen lassen, für die die jeweilige gesetzliche Regelung nicht gilt

Sicherstellung maximaler Vertraulichkeit/Nichteinsehbarkeit der Aktivitäten

Auswahl einer der gelisteten Organisation nahestehenden, aber nicht gelisteten Person als Verhandlungsführer*in

\begin{tabular}{ll}
\hline Beispielhaftes & Konditionierte Priorisierung: Imperative der Inklusivität und Ownership gehen \\
Vorgehen nach & vor, solange Risiko der Straffälligkeit von Mediationsakteuren mit durchgehender \\
Gesamtabwä- & juristischer Begleitung und ausgewogener Risikoverteilung minimiert wird \\
gung & $\begin{array}{l}\text { Konditionierte Sequenzierung: Mediation Support als Anreiz zur Waffenstillstands- } \\
\text { aushandlung verwenden }\end{array}$ \\
& $\begin{array}{l}\text { Kompartmentalisierung: breite Fächerung von Rollen und Handlungsschritten mit } \\
\text { transparenten Arbeitsschritten; Akzent auf Positiveffekte weitergeführter Gespräche } \\
\\
\text { in der Öffentlichkeit setzen }\end{array}$ \\
\hline
\end{tabular}

Sequenzierung, die Konfliktparteien in getrennten Gruppen simultan an normativen Forderungen arbeiten lässt bis die Vorschläge in der Gesamtgruppe angenommen werden (nothing is agreed until everything is agreed). Bei einer Kompartmentalisierung werden diejenigen Rollen und Maßnahmen, die sich nicht gleichzeitig konfliktfrei von einem Akteur ausüben lassen, auf verschiedene Akteure oder Prozesse aufgeteilt (AA und IMSD 2017, S. 13).

Für den Umgang mit Kollisionen innerhalb derselben normativen Sphäre empfiehlt sich das fallspezifische Ausbuchstabieren und Abwägen der jeweiligen Implikationen eines Normbruchs. Gewählt werden sollte schlicht die Verhaltensvariante 
Tab. 2 Fallbeispiel 2/Völkerrechtswidrige Annexion (in Anlehnung an Grafik in AA und IMSD 2017, S. 15)

\begin{tabular}{|c|c|c|}
\hline $\begin{array}{l}\text { Frage im Zen- } \\
\text { trum der Nor- } \\
\text { menkollision }\end{array}$ & \multicolumn{2}{|c|}{$\begin{array}{l}\text { Darf bzw. soll ein*e Mediator*in die Verletzung eines völkerrechtlichen Grund- } \\
\text { prinzips wie territoriale Unversehrtheit durch eine Konfliktpartei aus Friedensver- } \\
\text { handlungen ausklammern, um die Verhandlungsbereitschaft dieser Konfliktpartei zu } \\
\text { erhalten? }\end{array}$} \\
\hline $\begin{array}{l}\text { Relevante } \\
\text { Normen }\end{array}$ & \multicolumn{2}{|c|}{$\begin{array}{l}\text { Pro: Methodische, strategische, politische Imperative zum Erhalt der Verhandlungs- } \\
\text { bereitschaft }\end{array}$} \\
\hline & \multicolumn{2}{|c|}{$\begin{array}{l}\text { Contra: Völkerrechtliche Norm der territorialen Unversehrtheit von Staaten; evtl. } \\
\text { ethisches Prinzip der Allparteilichkeit }\end{array}$} \\
\hline \multirow{3}{*}{$\begin{array}{l}\text { Kosten-Nutzen- } \\
\text { Abwägung: } \\
\text { Beachtung vs. } \\
\text { Missachtung }\end{array}$} & $\begin{array}{l}\text { Pro: Erhalt der Verhandlungsbe- } \\
\text { reitschaft }\end{array}$ & $\begin{array}{l}\text { Contra: Territoriale Unversehrtheit von Staa- } \\
\text { ten }\end{array}$ \\
\hline & $\begin{array}{l}\text { Sicherung (statt Gefährdung) der } \\
\text { Bereitschaft der des Normbruchs } \\
\text { bezichtigten Konfliktpartei zur } \\
\text { weiteren Aushandlung/Einigung }\end{array}$ & $\begin{array}{l}\text { Wahrung (statt Verlust) des Vertrauens in } \\
\text { den Mediationsprozess auf Seiten des durch } \\
\text { Rechtsverletzung betroffenen Akteurs und Be- } \\
\text { wusstwerden der Geltung rechtlicher Grenzen } \\
\text { auf Seiten des rechtsverletzenden Akteurs }\end{array}$ \\
\hline & $\begin{array}{l}\text { Ausklammerung von (statt Über- } \\
\text { ladung mit und Entführung in) } \\
\text { Rechtsfragen zum Erhalt der } \\
\text { Arbeitsfähigkeit eines politisch } \\
\text { komplexen Mediationsprozesses }\end{array}$ & $\begin{array}{l}\text { Stärkung (statt impliziter Entkräftung) der } \\
\text { internationalen Rechtsordnung und der wahr- } \\
\text { genommenen Allparteilichkeit (statt Partei- } \\
\text { lichkeit des/der Mediator*in durch implizite, } \\
\text { einseitige Zugeständnisse an rechtsverletzende } \\
\text { Konfliktpartei) }\end{array}$ \\
\hline
\end{tabular}

Handlungs- $\quad$ Vermittlung unter transparenter Benennung divergierender Rechtsauffassungen bei-
möglichkeiten der Konfliktparteien (explizit nicht-evaluativer Mediationsansatz);

zur Ausbalancierung

Ausklammern der völkerrechtlichen Thematik im ersten Schritt (zeitliches Sequenzieren)

Anerkennung der Völkerrechtsverletzung zu Bedingung für Aufnahme von Vermittlungsprozess erklären, aber keine Thematisierung innerhalb der Vermittlung Delegation der Drittparteirolle an nicht an Völkerrecht gebundenen Akteur

Beispielhaftes Priorisierung: Respekt für beide Normensysteme schaffen (Betonung ggü. Parteien Vorgehen nach und Öffentlichkeit, dass Verhandlungen nicht im rechtsfreien Raum stattfinden), aber Gesamtabwä- Mediation explizit als Verfahren für Verhandlung von Interessens- nicht Rechtsnorgung men markieren

Kompartmentalisierung: Verteilung der mit Blick auf Normbruch-Sanktion notwendigen Rollen auf verschiedene Akteure (Mediator*in, UN), etwa parallele Initiierung rechtlicher Klärung durch IStGH

Agreement in Principle-Sequenzierung: Aufnahme der Thematik in (Zwischen-)Vereinbarungen als ungeklärter Themenkomplex, etwa als Memorandum, um stillschweigender normativer Anerkennung von geschaffenen Fakten entgegenzuwirken

mit den geringeren Folgekosten - es sollte also eine Priorisierung vorgenommen werden. Diese Folgekosten müssen innerhalb der jeweiligen Sphäre (etwa der Rechtsordnung) geprüft werden, aber auch jenseits davon. Zu diesen eher mittelbaren Kosten zählen etwa anfallende Belastungen für betroffene Individuen und Gruppen und die politischen Kosten bei bewusstem Bruch rechtlicher Vorgaben (AA und IMSD 2017, S. 13).

Zur tendenziell komplexeren Kollision zwischen Normen aus unterschiedlichen Sphären seien nachfolgend zwei Fallbeispiele angeführt, die zum einen die Sondierung von Verhandlungen mit als ,terroristisch“ bezeichneten Akteuren (Tab. 1), zum 
anderen den Umgang mit klar völkerrechtswidrigem Verhalten einer Konfliktpartei betreffen (Tab. 2).

Die Frage nach den normativen Grenzen und Spielräumen der Gesprächsführung mit Terrorist*innen zählt zu den regelmäßig umstrittenen Verfahrensfragen der Friedensmediation (Palmiano Federer 2019). Die Abwägung dieses Beispielfalls veranschaulicht: Ist man darauf vorbereitet, dass an vielen Stellen in Vermittlungsprozessen Zielkonflikte zwischen Methodik, Ethik und Recht in der Natur der Sache angelegt sind, kann das Vorgehen als Drittpartei gezielt darauf ausgerichtet werden.

Da es dabei maßgeblich um die Klärung und Eingrenzung des strafrechtlich sensiblen Bereichs für die konkrete Drittpartei geht, hier die Sachlage im Detail: Die Sicherheitsratsresolution 1373 (S/RES/1373 2001) vom 28. September 2001 verbietet in Nr. 2a unter Rückgriff auf Kapitel VII jede aktive oder passive Unterstützung für Terrorist*innen. Das Anbahnen und Durchführen einer Mediation mit als terroristisch eingestuften Gruppen durch staatliche Akteure stellt jedoch selbst bei weiter Deutung keine Unterstützung im Sinne der Resolution dar, verweist diese doch exemplarisch auf das Anwerben von Mitgliedern oder die Versorgung mit Waffen. Zu beachten ist dabei zudem, dass weder die Resolution noch sonstige völkerrechtliche Normen eine verbindliche und konsensfähige Definition des Terrorismus enthalten (Grozdanova 2014). Innerhalb nationaler „Antiterrorismusgesetze“ existieren allerdings höchst unterschiedliche - teils durchaus niedrigschwellige - Auslegungen dessen, was als Unterstützung von Terrorist*innen angesehen werden kann. Die USA etwa verfügen über eine Vielzahl an entsprechenden Sanktionsinstrumenten; die darin formulierten Verbote und Beschränkungen sind regelmäßig auch strafrechtlich relevant (Less 2004). Während also denkbar ist, dass private Akteure nach nationalen Gesetzgebungen für Vermittlungstätigkeiten unter Einbezug terroristisch eingestufter Gruppen strafrechtlich belangt werden, trifft dies für staatliche Akteure nicht $\mathrm{zu}$, da diese Strafrechtsordnungen prinzipiell nicht für staatliche Akteure gelten. Insofern kann die rechtliche Zulässigkeit von Mediationsaktivitäten unter aktiver Beteiligung terroristischer Gruppen nur anhand nationaler Gesetze bestimmt werden. Völkerrechtlich ist sie zulässig und damit aus staatlicher Sicht politisch vor dem Hintergrund der aktuellen Sensibilität und Relevanz des Themas entlang der oben skizzierten Kategorien abzuwägen.

Das zweite Fallbeispiel erweitert die Abwägung um die (außen)politische Dimension. Es verdeutlicht dabei die Sequenzierung und Rollentrennung als probate Reaktionsmöglichkeit (Tab. 2).

Eine der wohl zentralsten Normen der UN Charta, die als Reaktion auf das Scheitern des Völkerbundes sowie den Verlauf des Zweiten Weltkriegs zu sehen ist, stellt das Verbot von ,gegen die territoriale Unversehrtheit oder die politische Unabhängigkeit eines Staates gerichtete(r) oder sonst mit den Zielen der Vereinten Nationen unvereinbare(r) Androhung oder Anwendung von Gewalt" dar (Vereinte Nationen 1945). Dabei handelt es sich um eine Norm des zwingenden Völkerrechts, was ihre Relevanz für die Mediation noch einmal steigert (Dörr und Randelzhofer 2012). Viele bewaffnete Konflikte (Pettersson et al. 2019) und internationale Spannungslagen haben Auseinandersetzungen über Territorium zum Gegenstand. Handelt es sich dabei um einen Konflikt zwischen zwei Staaten, in dem einer der beiden Territorium entgegen Art. 2 (4) UN Charta annektiert hat, bedarf dieser Umstand der besonde- 
ren Aufmerksamkeit der Mediator*in. Auch hier zeigt die differenzierte Abwägung, dass durch Methoden wie Sequenzierung und Kompartmentalisierung, also eine Verteilung der mit Blick auf die Normbruch-Sanktion maßgeblichen Schritte auf der Zeitleiste oder aber auf mehrere Akteure, ein gleichermaßen normativ sensibles wie pragmatisches Vorgehen möglich ist. Es verlangt allerdings, den jeweiligen Kosten der verschiedenen Handlungsoptionen direkt ins Antlitz zu sehen.

\section{Fazit und Ausblick}

Dieser Artikel möchte den beiden Positionen, die den Diskurs zur Rolle von Normen in der Friedensmediation bislang prägen - der deutlichen Forderung nach normenorientierter Friedensmediation auf der einen und der Warnung vor normativer Überfrachtung auf der anderen Seite -, in Form einer Synthese entgegentreten.

Die normativen Herausforderungen, die mit der Friedensmediation in mehrfacher Hinsicht einhergehen, sind immens. In Vermittlungsprozessen können die Ordnungsrahmen von Recht, Ethik, Methodik und Politik massiv und entsprechend folgenschwer kollidieren. Ohne ein Ausbuchstabieren von normativen Zielkonflikten und ohne das sorgfältige Abwägen der Folgen der jeweiligen Verhaltensoptionen sind gute Prozesse, Verfahrensentscheidungen und Vereinbarungen schwer vorstellbar.

Die daraus resultierenden Dilemma-Situationen können dabei auch konstruktiv, nämlich als zentrale Weggabelungen verstanden werden, an denen sich die Weichen für ganze Friedensprozesse stellen können. Erkennt und benennt man die Normen mitsamt der durch sie hervorgebrachten Spannungen und Zielkonflikte, dienen sie als hilfreiche Indikatoren für gute und bewusste Entscheidungen in Mediationsprozessen. Gerade die normativen Sondersituationen, die durch das vielschichtige Zusammenwirken zwischen staatlichen und nicht-staatlichen Vermittler*innen und Konfliktparteien entstehen, können durch eine solide normative Perspektive genauer erfasst und besser gehandhabt werden. Denn mit der Komplexität wachsen glücklicherweise gleichzeitig die Handlungsoptionen in der Antwort auf diese Zielkonflikte. Normen lassen sich so, unvoreingenommen und in aller Relativität betrachtet, praktisch als das Rückgrat guter Lösungen nutzen: sie definieren die sicheren Spielräume auf dem langen Weg zu nachhaltigen Vereinbarungen.

Danksagung Die Autor*innen danken Tanja Rollett und Anna Dick für das sorgfältige inhaltliche und formale Lektorat.

Funding Open Access funding enabled and organized by Projekt DEAL.

Open Access Dieser Artikel wird unter der Creative Commons Namensnennung 4.0 International Lizenz veröffentlicht, welche die Nutzung, Vervielfältigung, Bearbeitung, Verbreitung und Wiedergabe in jeglichem Medium und Format erlaubt, sofern Sie den/die ursprünglichen Autor(en) und die Quelle ordnungsgemäß nennen, einen Link zur Creative Commons Lizenz beifügen und angeben, ob Änderungen vorgenommen wurden.

Die in diesem Artikel enthaltenen Bilder und sonstiges Drittmaterial unterliegen ebenfalls der genannten Creative Commons Lizenz, sofern sich aus der Abbildungslegende nichts anderes ergibt. Sofern das betreffende Material nicht unter der genannten Creative Commons Lizenz steht und die betreffende Handlung nicht nach gesetzlichen Vorschriften erlaubt ist, ist für die oben aufgeführten Weiterverwendungen des Materials die Einwilligung des jeweiligen Rechteinhabers einzuholen. 
Weitere Details zur Lizenz entnehmen Sie bitte der Lizenzinformation auf http://creativecommons.org/ licenses/by/4.0/deed.de.

\section{Einhaltung ethischer Richtlinien}

Interessenkonflikt A. Holper, L. Kirchhoff und F. Würkert geben an, dass kein Interessenkonflikt besteht.

Ethische Standards Für diesen Beitrag wurden von den Autoren keine Studien an Menschen oder Tieren durchgeführt. Für die aufgeführten Studien gelten die jeweils dort angegebenen ethischen Richtlinien.

\section{Literatur}

ACCORD, African Union. 2014. African union mediation support handbook. https://www.accord.org.za/ publication/african-union-mediation-support-handbook. Zugegriffen: 25. Mai 2020.

Acharya, Amitav P.A.R.T.I.C.L.E.D.O.T. 2004. How ideas spread. Whose norms matter? Norm localization and institutional change in Asian regionalism. International Organization 58(2):239-275.

Arnault, Jean. 2014. Legitimacy and peace processes. International norms and local realities. Accord 2014(25):21-25.

Auswärtiges Amt (AA), und Initiative Mediation Support Deutschland (IMSD). 2017. Normativer Bezugsrahmen und völkerrechtliche Grundlagen der Friedensmediation. Fact Sheet-Reihe: Friedensmediation und Mediation Support 5. https://www.auswaertiges-amt.de/blob/1792316/ 10398297f5a3a14bdc2eb6efb56abc26/bezugsrahmen-data.pdf. Zugegriffen: 25. Mai 2020.

Barnett, Michael N., und Martha Finnemore. 2004. Rules for the world. International organizations in global politics. Ithaca: Cornell University Press. https://doi.org/10.7591/j.ctt7z7mx.

Binder, Christina. 2011. Auf dem Weg zum lateinamerikanischen Verfassungsgericht? Die Rechtsprechung des Interamerikanischen Menschenrechtsgerichtshofs im Bereich der Amnestien. Zeitschrift für ausländisches öffentliches Recht und Völkerrecht 2011(71):1-29.

Björkdahl, Annika. 2002. Norms in international relations. Some conceptual and methodological reflections. Cambridge Review of International Affairs 15(1):9-23. https://doi.org/10.1080/095575702201 26216.

Breidenbach, Stephan. 2005. Vermittlung von Recht im Konflikt. Grenzüberschreitungen. In Beiträge zum internationalen Verfahrensrecht und zur Schiedsgerichtsbarkeit, Hrsg. Birgit Bachmann, Stephan Breidenbach, Dagmar Coester-Waltjen, Burkhard Heß, Andreas Nelle und Christian Wolf, 83-95. Tübingen: Mohr Siebeck.

Coady, Cecil A.J. 2014. The problem of dirty hands. https://plato.stanford.edu/entries/dirty-hands. Zugegriffen: 25. Mai 2020.

Coleman, Stephen. 2015. Even dirtier hands in war. Considering Walzer's supreme emergency argument. In Conscience, leadership and the problem of 'dirty hands, Hrsg. Matthew Beard und Sandra Lynch, 61-73. Bradford: Emerald Group Publishing Limited.

de Coning, Cedric. 2018. Adaptive Peacebuilding. International Affairs 94(2):301-317.

Convergne, Elodie. 2016. Learning to mediate? The mediation support unit and the production of expertise by the UN. Journal of Intervention and Statebuilding 10(2):181-199. https://doi.org/10.1080/ 17502977.2015.1079959.

Council of the European Union. 2009. Concept on strengthening EU mediation and dialogue capacities. http://www.eeas.europa.eu/archives/docs/cfsp/conflict_prevention/docs/concept_strengthening_ eu_med_en.pdf. Zugegriffen: 25. Mai 2020.

Dancy, Geoff, und Eric Wiebelhaus-Brahm. 2017. The impact of criminal prosecutions during intrastate conflict. Journal of Peace Research 55(1):47-61. https://doi.org/10.1177/0022343317732614.

Dörr, Oliver, und Albrecht Randelzhofer. 2012. Article 2 (4). In The charter of the United nations. A commentary, 3. Aufl., Hrsg. Bruno Simma, Daniel-Erasmus Khan, Georg Nolte, Andreas Paulus und Nikolai Wessendorf, 200-234. Oxford: Oxford University Press.

European Union External Action Service. 2012. Factsheet-EEAS mediation support project—knowledge product transitional justice in the context of peace mediation. http://www.europarl.europa.eu/ meetdocs/2009_2014/documents/droi/dv/401_factsheettransjust_/401_factsheettransjust_en.pdf. Zugegriffen: 25. Mai 2020.

Finnemore, Martha. 2000. Are legal norms distinctive? New York University Journal of International Law and Politics 32(3):699-706. 
Grozdanova, Rumyana. 2014. 'Terrorism' - too elusive a term for an international legal definition? Netherlands International Law Review 61(3):305-334. https://doi.org/10.1017/S0165070X14001351.

Hart, Herbert L.A. 1994. The concept of law, 2. Aufl., Oxford, New York: Clarendon Press; Oxford University Press.

Hellmüller, Sara, und Marie-Joëlle Zahar. 2019. UN-led mediation in Syria and civil society. Accord 2019(28):84-87.

Hellmüller, Sara, Julia Palmiano Federer, und Matthias Zeller. 2015. The role of norms in international peace mediation. http://www.swisspeace.ch/. Zugegriffen: 25. Mai 2020.

Hellmüller, Sara, Julia Palmiano Federer, und Jamie Pring. 2017. Are mediators norm entrepreneurs? Exploring the role of mediators in norm diffusion. swisspeace working papers 3. Bern: swisspeace.

Henckaerts, Jean-Marie, und Cornelius Wiesener. 2020. Human rights obligations of non-state armed groups. In International humanitarian law and non-state actors, Hrsg. Ezequiel Heffes, Marcos D. Kotlik und Manuel J. Ventura, 195-227. Den Haag: T.M.C. Asser Press.

Holper, Anne, und Lars Kirchhoff (Hrsg.). 2020. Friedensmediation - Spannungsfeld aus Methodik, Macht und Politik. Reihe Interdisziplinäre Studien zu Mediation und Konfliktmanagement. Baden-Baden: Nomos.

International Law Commission. 2018. Draft conclusions on identification of customary international law, with commentaries. In Yearbook of the international law commission, Hrsg. International Law Commission, 122-156. New York. http://legal.un.org/docs/?path=./ilc/texts/instruments/english/ commentaries/1_13_2018.pdf\&lang=EF. Zugegriffen: 25. Mai 2020.

International Law Commission. 2019. Peremptory norms of general international law (jus cogens). Text of the draft conclusions and Draft Annex Provisionally adopted by the Drafting Committee on First Reading (A/CN.4/L.936). http://legal.un.org/docs/?symbol=A/CN.4/L.936. Zugegriffen: 25. Mai 2020 .

Joinet, Louis. 1997. Question of the impunity of perpetrators of human rights violations. Revised final report prepared by the Special Rapporteur of the U.N. Sub-Commission on the Prevention of Discrimination and the Protection of Minorities, E/CN.4/Sub.2/1997/20/Rev.1. https://documents-dds-ny.un. org/doc/UNDOC/GEN/G97/141/42/pdf/G9714142.pdf?OpenElement. Zugegriffen: 25. Mai 2020.

Kastner, Philipp. 2015. Legal normativity in the resolution of internal armed conflict. New York: Cambridge University Press.

Katzenstein, Peter J. 1996. Introduction. Alternative perspective on national security. In The culture of national security. Norms and identity in world politics, Hrsg. Peter Katzenstein, 1-27. New York: Columbia University Press.

Kirchhoff, Lars. 2008. Constructive interventions. Paradigms, process and practice of international mediation. Alphen aan den Rijn: Kluwer Law International.

Kirchhoff, Lars. 2009. Linking mediation and transitional justice. The use of interest-based mediation in processes of transition. Building a future on peace and justice. Studies on transitional justice, peace and development. In The Nuremberg declaration on peace and justice, Hrsg. Kai Ambos, Judith Large, und Marieke Wierda, 237-262. Berlin: Springer.

Kolb, Robert. 2015. Peremptory international law-jus Cogens. A general inventory. London: Bloomsbury publishing.

Kraus, jetzt Holper, Anne I. 2011. Interkulturelle Verfahrensethik. Ein Modell zur Vermittlung von Konflikten zwischen partikularen Verfahrensnormen. Stuttgart: Kohlhammer.

Kraus, jetzt Holper, Anne I., Owen Frazer, Lars Kirchhoff, Tatiana Kyselova, Simon Mason, und Julia Palmiano Federer. 2019. Dilemmas and trade-offs in peacemaking. A framework for navigating difficult decisions. Politics and Governance https://doi.org/10.17645/pag.v7i4.2234.

Less, Steven. 2004. Country report on the USA. In Terrorism as a challenge for national and international law: security versus liberty?, Hrsg. Christian Walter, Silja Vöneky, Volker Röben und Frank Schorkopf, 633-732. Berlin: Springer.

Luhmann, Niklas. 1987. Rechtssoziologie, 3. Aufl., Opladen: Westdeutscher Verlag.

Mancini, Francesco, und Jose Vericat. 2016. Lost in transition. UN mediation in Libya, Syria, and Yemen. International peace institute. https://papers.ssrn.com/sol3/papers.cfm?abstract_id=2883306. Zugegriffen: 25. Mai 2020.

Mason, Simon. 2016. Combining best, good, and emergent practice. nadelNEWS 2016(1):3-4.

McAdams, Richard H. 1997. The origin, development, and regulation of norms. Michigan Law Review 96(2):338-433.

Mediation Support Network (MSN). 2012. Translating mediation guidance into practice. http://peacemaker. un.org/sites/peacemaker.un.org/files/TranslatingMediationGuidanceIntoPractice_MSN_2012.pdf. Zugegriffen: 25. Mai 2020. 
Mediation Support Network. 2013. Translating mediation guidance into practice. Commentary on the UN guidance for effective mediation. http:/www.css.ethz.ch/content/dam/ethz/special-interest/gess/ cis/center-for-securities-studies/pdfs/Discussion_Points_2_Translating_Mediation_Guidance_into_ Practice.pdf. Zugegriffen: 25. Mai 2020.

Möllers, Christoph. 2015. Die Möglichkeit der Normen. Über eine Praxis jenseits von Moralität und Kausalität. Berlin: Suhrkamp.

Nilsson, Desirée. 2008. Partial peace. Rebel groups inside and outside of civil war settlements. Journal of Peace Research 45(4):479-495. https://doi.org/10.1177/0022343308091357.

Nilsson, Desirée. 2012. Anchoring the peace. Civil society actors in peace accords and durable peace. International Interactions 38(2):243-266. https://doi.org/10.1080/03050629.2012.659139.

OSCE. 2014. Mediation and dialogue facilitation. Reference guide. http://www.osce.org/secretariat/ 126646?download=true. Zugegriffen: 25. Mai 2020.

Palmiano Federer, Julia. 2016. Navigating international norms in peace mediation. https://voelkerrechtsblog. org/navigating-international-norms-in-peace-mediation. Zugegriffen: 25. Mai 2020.

Palmiano Federer, Julia. 2019. We do negotiate with terrorists: navigating liberal and illiberal norms in peace mediation. Critical Studies on Terrorism 12(1):19-39. https://doi.org/10.1080/17539153.2018. 1472727.

Pettersson, Therése, Stina Högbladh, und Magnus Öberg. 2019. Organized violence, 1989-2018 and peace agreements. Journal of Peace Research 56(4):589-603. https://doi.org/10.1177/0022343319856046.

Paffenholz, Thania. 2018. Women in peace negotiations. In Gendering diplomacy and international negotiation, Hrsg. Karin Aggestam, Ann E. Towns, 169-191. Cham: Springer.

Piiparinen, Touko. 2013. Law versus bureaucratic culture. The case of the ICC and the transcendence of instrumental rationality. Normative pluralism and international law. In Exploring Global Governance, Hrsg. Jan Klabbers und Touko Piiparinen, 251-283. Cambridge: Cambridge University Press.

Reckwitz, Andreas. 2000. Die Transformation der Kulturtheorien: zur Entwicklung eines Theorieprogramms. Weilerswist: Velbrück Wissennschaft.

Rodman, Kenneth A. 2009. Is peace in the interests of justice? The case for broad prosecutorial discretion at the international criminal court. Leiden Journal of International Law 22(1):99-126.

Sieckmann, Jan-Reinard. 2018. Rechtsphilosophie. Tübingen: Mohr Siebeck.

Song, Lili. 2019. Strengthening responsibility sharing with south—south cooperation. China's role in the global compact on refugees. International Journal of Refugee Law 30(4):687-690. https://doi.org/10. 1093/ijrl/eey059.

de Souza Dias, Talita. 2017. 'Interests of justice'. Defining the scope of prosecutorial discretion in article $53(1)(c)$ and $(2)(c)$ of the rome statute of the international criminal court. Leiden Journal of International Law 30(3):731-751. https://doi.org/10.1017/S092215651700022X.

Svensson, Isak. 2007. Mediation with muscles or minds? Exploring power mediators and pure mediators in civil wars. International Negotiotion 12(2):229-248. https://doi.org/10.1163/138234007X223294.

Tisdall, Simon. 2018. UN tells of Myanmar genocide but are world powers listening? The guardian, 27.08.2018. https://www.theguardian.com/world/2018/aug/27/un-claim-of-myanmar-genocide-isa-litmus-test-for-global-justice. Zugegriffen: 25. Mai 2020.

Urueña, René. 2017. Prosecutorial politics. The ICC's influence in Colombian peace processes 2003-2017. Am. J. Int. Law 111(1):104-125. https://doi.org/10.1017/ajil.2016.3.

Vereinte Nationen (Office of Legal Affairs. Codification Division). 1992. Handbook on the peaceful settlement of disputes between states. New York: Vereinte Nationen.

Vereinte Nationen. 1945. United Nations Charta. San Francisco. https://www.unric.org/de/charta. Zugegriffen: 25. Mai 2020.

Vereinte Nationen. 1982. A/RES/37/10, Manila declaration on the peaceful settlement of disputes. https://peacemaker.un.org/sites/peacemaker.un.org/files/GARES_ManilaDeclaration_ARES3710 \%28english\%29_0.pdf. Zugegriffen: 13. Jan. 2021.

Vereinte Nationen. 2012. UN guidance or effective mediation. New York, NY. https://peacemaker.un. org/sites/peacemaker.un.org/files/GuidanceEffectiveMediation_UNDPA2012\%28english\%29_0.pdf. Zugegriffen: 25. Mai 2020.

Wählisch, Martin. 2016. Normative limits of peace negotiations: questions, guidance and prospects. Global Policy 7(2):261-266. https://doi.org/10.1111/1758-5899.12325.

Waldman, Ellen. 2011. Mediation ethics. Cases and commentaries. Hoboken: Wiley \& Sons.

Walzer, Michael. 1986. Supreme emergency. War, morality, and the military profession, 2. Aufl., 425-441. Boulder: Westview Pr.

Weiss, Joshua N., und Sarah Rosenberg. 2003. Sequencing strategies and tactics. Beyond intractability. https://www.beyondintractability.org/essay/issue-segmentation. Zugegriffen: 25. Mai 2020. 
Wiener, Antje. 2007. The dual quality of norms and governance beyond the state: sociological and normative approaches to interaction. Critical Review of International Social and Political Philosophy 10(1):47-69. https://doi.org/10.1080/13698230601122412.

Wiener, Antje, und Uwe Puetter. 2009. The quality of norm is what actors make of it. Critical constructivist research on norms. (special issue: contested norms in international relations). Journal of International Law and International Relations 5(1):1-16.

Würkert, Felix. 2021. Rechtsnormen und Legitimität in der Friedensmediation. Dissertationsschrift. Eingereicht 2021. Frankfurt (Oder): Europa-Universität Viadrina.

Zeller, Matthias, und Hein Schellekens. 2017. On democracy. The role of norms in international peace mediation. https://www.swisspeace.ch/fileadmin/user_upload/Media/Publications/Essentials/SP_ Essential_1703-WEB.pdf. Zugegriffen: 25. Mai 2020. 